\title{
The Super-Kamiokande experiment
}

\author{
Yoichiro Suzuki $^{\mathrm{a}}$ \\ Kamioka Satellite, Kavli Institute for the Physics and Mathematics of the Universe (WPI), The University of Tokyo, 246 Higashi-Mozumi, \\ Kamioka, Hida 506-1205, Japan
}

Received: 12 March 2019 / Accepted: 18 March 2019 / Published online: 2 April 2019

(C) The Author(s) 2019

\begin{abstract}
Super-Kamiokande is a gigantic and versatile detector able to detect neutrinos with energies between a few $\mathrm{MeV}$ and a few hundred GeV. Super-K started data taking on 1st of April in 1996 after 5 years construction period and obtained compelling evidence of atmospheric neutrino oscillations in 1998, shortly after the beginning of the experiment. In 2001 SNO in Canada together with the Super-K data established that solar neutrinos are also oscillating. Following those historical discoveries, numerous intriguing results have been obtained by Super-K, like the discovery of oscillatory behavior, tau appearance in the atmospheric neutrinos, the matter effect of the solar neutrinos through the earth. The Super-K detector has also been used as a far detector of the long baseline neutrino oscillation experiments, $\mathrm{K} 2 \mathrm{~K}$ and $\mathrm{T} 2 \mathrm{~K}$. In this article, we report mostly on the studies of the neutrino oscillations by Super-K in a historical context. The prospects for the future of Super-K are also described.
\end{abstract}

\section{Contents}

1 Introduction - historical overview . . . . . . . . 1

2 Detector and characteristics ... . . . . . . . 3

3 Neutrino oscillations . . . . . . . . . . . . 5

4 Atmospheric neutrinos . . . . . . . . . . . 5

5 The discovery of neutrino oscillation in $1998 \ldots 8$

6 Current situation of the atmospheric neutrinos . . . 8

7 Solar Neutrinos . . . . . . . . . . . . . . . . . . 10

8 Discovery of the solar neutrino oscillation . . . . . 14

9 Current situation of the solar neutrinos . . . . . . 15

10Summary and future . . . . . . . . . . . 17

References . . . . . . . . . . . . . . . 18

a e-mail: yoichiro.suzuki@ipmu.jp

\section{Introduction - historical overview}

Super-Kamiokande (Super-K, hereafter), the world largest imaging water Cherenkov detector, has been operated for more than 20 years since 1996, performed detailed studies on neutrino properties, and eventually led to the discovery of neutrino oscillations opening up a new field of research. This report describes the history and the physics results of the Super-K experiment. We first refer to "Kamiokande" briefly, the predecessor of Super-K, as a prehistory. Much of the historical information written here about Kamiokande comes from the recollections of the Kamiokande collaborators and references. Some of them are written in Japanese [1,2].

The beginning of the story goes back to the middle of 70's when particle physicists had started to discuss their dream to unify the weak, electromagnetic and strong interactions, the gauge group of $\mathrm{SU}(2) \times \mathrm{U}(1) \times \mathrm{SU}(3)$, by a single larger gauge group. In 1974, Georgi and Glashow [3] presented a first realistic model of the grand unification based upon $\mathrm{SU}(5)$. They said in the paper that "It makes just one easily testable prediction, $\sin ^{2} \theta_{w}=\frac{3}{8}$. It also predicts that the proton decays - but with an unknown and adjustable rate". Soon after, Georgi, Quinn and Weinberg [4] showed a more specific estimate of a proton lifetime, $\tau_{p}=6 \times 10^{31}$ years, for the superheavy gauge boson mass of $\mathrm{M}_{\mathrm{X}}=5 \times 10^{15} \mathrm{GeV}$. The best experimental lower limit of the proton lifetime at that time in 1974 was $2 \times 10^{30}$ years [5] using 20 tons of liquid scintillator to look for proton decay into muons which were identified in coincidence with the $\mu \rightarrow$ e decay sequence. Experimentalists thought that proton decay was within reach of experimental searches since 1000 tons of water contains $\sim 6 \times 10^{32}$ nucleons and a race with underground experiments started. Nevertheless, we now know that the estimated lifetime was considerably underestimated.

Koshiba and his colleagues conceived to build a detector of about 2000 tons of water (inner volume) surrounded by $\sim 1000$ PMTs (photo-multiplier tubes) of $50 \mathrm{~cm}$ in diameter to look for proton decay [1] to test grand unified the- 
ories. In 1982, the project called KamiokaNDE (Kamioka Nucleon Decay Experiment) was funded. Although the primary aim was to conduct an extensive search for proton decay, possibilities to make a study on neutrino oscillations through atmospheric neutrinos and to detect neutrino bursts from supernovae were mentioned in their proposal, however a possible observation of solar neutrinos was not explicitly referred [2].

In July, 1983, the Kamiokande experiment started to take data while the competitor, the IMB experiment, using $\sim 8000$ tons of water with $\sim 5000$ PMTs of $20 \mathrm{~cm}$ in diameter had already started 1 year before. The target mass of IMB exceeded significantly the one of Kamiokande. However, Kamiokande was expected to achieve higher energy resolution and lower energy threshold, since $20 \%$ of the inner surface was covered by light sensitive photo-cathode, while IMB PMTs covered $2 \%$.

\section{From Kamiokande to Super-Kamiokande}

A few months after the start of Kamiokande, they had realized that they could observe electrons from muon decay down to $15 \mathrm{MeV}$ and recognized that further efforts to lower the detectable energy down to $10 \mathrm{MeV}$ would make it possible to measure solar neutrinos. Solar neutrinos became an important subject for Kamiokande. In 1984, at ICOBAN84 held in Park City, Utah, the Kamiokande collaboration made two presentations, one was the report on their latest physics results and a possible detector improvement aiming at observing solar neutrinos [6] and another one was a proposal to construct a $22.5 \mathrm{kton}$ water Cherenkov detector called JACK (Japan America Collaboration at Kamioka) [7]. It was called Super-Kamiokande (Super-K) soon after their initial naming.

Responding to the proposed detector improvement, a US group (mostly from University of Pennsylvania) joined and the new Collaboration, Kamiokande-II, was formed. New TDC modules were arranged by the US group. An anticounter was newly installed and a water circulation system was introduced. After fighting against the low energy backgrounds mostly from the Rn contamination in water, the experiment had succeeded to lower the energy threshold. Kamiokande-II started in early 1987, and immediately after that the historical observation of the neutrino burst from supernova SN1987A [8] was made, which demonstrated the excellent capability of water Cherenkov detectors to measure low energy neutrinos. A couple of years later Kamiokande-II also had succeeded to detect solar neutrinos and confirmed the deficit of neutrinos from the sun [9].

It is interesting to note what kind of physics goals were addressed or written in the early Super-K proposals that were presented in late 80's. The situation of the solar neutrinos and the atmospheric neutrinos had been greatly changing during that time, which had affected the proposals of
Super-K in those years. In the early times of the Super-K proposal in 1986, proton decay was a top priority subject of the project, then the neutrino astronomy was extensively added for the 1987 revision due to the observation of the neutrino burst, which definitely worked as a strong back up for the planned project. When the construction of Super-K was approved in 1991, the top listed subjects of Super-K was the neutrino astronomy, solar neutrinos and supernova neutrinos, and proton decay. The atmospheric neutrino anomaly indicated in 1988 was still under debate. The importance of the atmospheric neutrinos had been increasing even during the construction of the detector between 1991 and 1996. The construction of the Super-K detector was completed in 1996. When Super-K started, not only the solar neutrino study and the search for proton decay, but also the atmospheric neutrinos became one of the important subjects of Super-K.

\section{Super-Kamiokande}

The new and largest neutrino detector, Super-Kamiokande (Sect. 2) was expected to give answers to those neutrino problems. The event rate per day in its $22 \mathrm{kt}$ fiducial mass were supposed to be $\sim 10$ and $\sim 15$ observable interactions for atmospheric and solar neutrinos, respectively, with $4.5 \mathrm{MeV}$ (kinetic) energy threshold. With this high statistics measurement, we anticipated to obtain model independent evidence of solar neutrino oscillations, namely the energy spectrum distortion, a time variation and so on. Precise measurements of the asymmetry of the zenith angle distribution of the atmospheric neutrinos would directly demonstrate the existence of neutrino oscillations (Sect. 3).

People thought that the atmospheric and solar neutrino problems might be resolved soon after the start of Super-K. In fact the discovery of neutrino oscillation (Sects. 4, 5) was announced in 1998 in the study of the atmospheric neutrinos by Super-K 2 years after the start, while the evidence of the solar neutrino oscillation was obtained by comparing two data sets from Super-K and SNO in 2001 (Sects. 7, 8). They were two big milestones of Super-K.

The first earth-scale long baseline neutrino oscillation experiment, K2K (KEK to Kamioka) starting in June 1999, confirmed the atmospheric neutrino oscillation, and in 2002, KamLAND [10], the long baseline reactor experiment, confirmed the solar neutrino oscillation and examined the oscillation parameters. The neutrino oscillation became the major topic in the particle physics resulting in strong research programs.

Super-K has continued in producing important physics results subsequent to the two important discoveries. Super-K observed the oscillatory behavior in atmospheric neutrinos, confirmed the appearance of tau neutrinos, and showed implications of neutrino mass hierarchy and non zero $\mathrm{CP}$ phase (Sect. 6). Super-K revealed the matter effect on the neutrino 


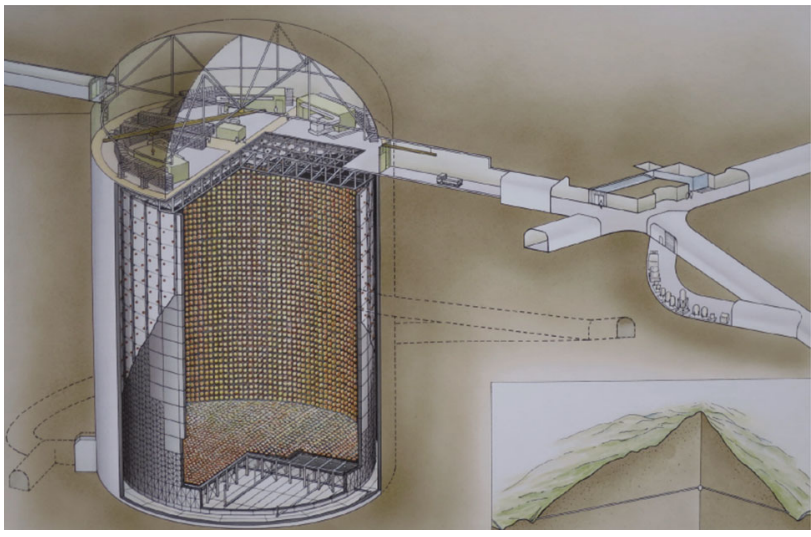

Fig. 1 Super-Kamiokande Detector. The $50 \mathrm{kton}$ water is viewed by 11, 000 photomultiplier tubes (PMT) and placed $1000 \mathrm{~m}$ underground

oscillation through the day/night flux difference of the solar neutrinos and was exploring the upturn of the solar neutrino spectrum (Sect. 9). Super-K is still very active, even 20 years after the start of the experiment.

The Super-K collaboration consists of about 175 physicists from 44 institutions over 10 countries (as of 2018).

\section{Detector and characteristics}

Super-Kamiokande is located $1000 \mathrm{~m}$ underground in the Kamioka mine, Gifu prefecture, Japan. The horizontal entrance tunnel leads us to the experimental area through $1.7 \mathrm{~km}$ drive, which allows us to access the detector for $24 \mathrm{~h}$ for maintenance.

Super-K is a cylindrically shaped detector with $42.2 \mathrm{~m}$ in height and $39.6 \mathrm{~m}$ in diameter containing $50 \mathrm{kton}$ of water inside as shown in Fig. 1. The inner $32 \mathrm{kton}$ (ID) is surrounded by about eleven thousand photomultiplier tubes of $50 \mathrm{~cm}$ diameter covering by their photo-cathode $40 \%$ of the inner surface. Its fiducial mass is 22.5 ktons where the outer edge of this volume is located $2 \mathrm{~m}$ inside of the PMT surface plane.

The inner detector is surrounded by the outer detector (OD) of $\sim 2$ to $3 \mathrm{~m}$ thick water layers viewed by 1885 PMTs of $20 \mathrm{~cm}$ diameter, which are used to shield and identify incoming particles.

Super-K was funded in 1991 and its construction took 5 years. It was just 4 years after the historical observation of the neutrino burst from the supernova in our adjacent galaxy. In 1992, 1 year after the start of the construction of the detector, a US group who had been working on the IMB experiment had joined the Super-K project. They took the responsibility to fabricate an outer detector system including photo-sensors. The excavation of the cavity for the detector finished in June 1994. The stainless steal water tank had been constructed from June 1994 to June 1995. It took about 6 months to install

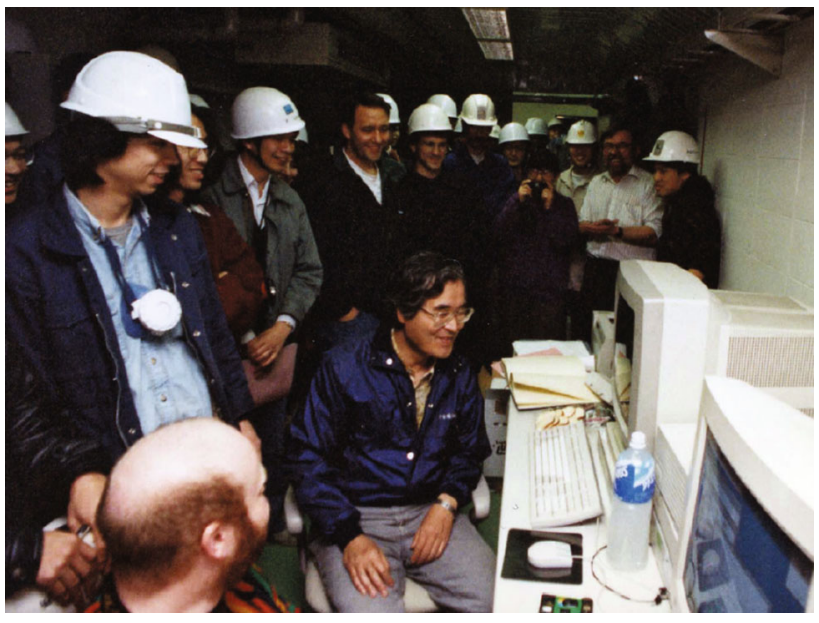

Fig. 2 A photograph taken at 0:00 on April 1st, 1996, the start of the Super-Kamiokande eperiment

the photomultiplier tubes (PMTs), electronics and data acquisition system. We had started to fill the detector with water in January, 1996. Figure 2. shows the moment when Yoji Totsuka pressed the button to start the experiment punctually at 0:00 on April 1st, 1996.

After the completion of the initial phase (SK-I) of the data taking in 2001, we had drained the water and replaced hundreds of electrically defected PMTs. During the time of filling the water subsequent to the replacement of those PMTs, we had a tragic accident leading to a loss of 6777 out of 11,146 PMTs through a chain reaction of implosions transmitted by shock waves contiguously created by the adjacent implosions. This accident arose by one of the PMTs arranged at the bottom of the water tank. We had cleaned up the detector and re-distributed PMTs that remained in our hands. The number of total PMTs used in the detector after the accident was roughly half. Super-K restarted as SK-II at the end of the year 2002. This phase with smaller number of PMTs had continued for about 3 years. Then, in 2005, the full restoration work had been conducted. SK-III equipped with 11,134 inner PMTs started to take data in July 2006 and the current phase of the detector, SK-IV has been running and stably taking data since September, 2008. See Table 1 for the details of the running phases of Super-K.

The 1st generation front end electronics called ATM (Ana$\log$ and Timing Module) was used for SK-I,II, and III and then replaced by a new electronics system, called QBEE (QTCBased Electronics with Ethernet). The new system has been continuously operating since September 2008 at the beginning of SK-IV. The old electronics system based on the PMT hit-trigger where those events that exceeded the threshold number of hit PMTs within 200 ns were recorded. Subsequently, the pulse height and the time were digitized by the analog to digital converters as described elsewhere [11] 
Table 1 Running phases of Super-Kamiokande and total number of data accumulated

\begin{tabular}{lllll}
\hline Phase & SK-I & SK-II & SK-III & SK-IV (as of 14-Feb) \\
\hline Periods & 96-Apr & 02-Dec & 06-Jul & Total \\
& $\sim$ 01-Jun & $\sim 05-$-Nov & $\sim 08$-Sep & $\sim$ running \\
ID PMT \# & $11,146(40 \%)$ & $5182(19 \%)$ & $11,129(40 \%)$ & $11,129(40 \%)$ \\
Electronics & ATM & ATM & ATM & QBEE \\
Trigger & Hardware & Hardware & Hardware & Software \\
Atm $v$ FC+PC & 1489 days & 799 days & 518 days & $(1417$ days $)$ \\
(\# of events) & $12,299+902$ & $6,610+427$ & $4,355+344$ & $(11,513+960)$ \\
& & & & $(1417$ days $)$ \\
Atm $v$ Up- $v$ & 1646 days & 828 days & 636 days & $(2085)$ \\
\# of events) & 2328 & 2094 & 945 & $(1693$ days $)$ \\
Solar $v$ & 1496 days & 791 days & 547 days & $(19,809.4)$ \\
$($ \# of events) & 22,404 & 7212.8 & 8147.0 & $(46.5 \mathrm{kt} *$ years $)$ \\
Proton decay & 91.7 kt*year & $49.2 \mathrm{kt} *$ year & $31.9 \mathrm{kt} *$ year & 4527 days \\
\hline
\end{tabular}

The new electronics, QBEE, recorded every hit of all the PMTs including the PMT's dark current, typically a few $\mathrm{kHz}$ for each PMT. A software trigger extracts an event from the recorded hit information and provides another handling of lowering the threshold and making up a sophisticated trigger. This is the most prominent feature of the new electronics. A single QBEE board has 24 input channels and 472 modules for the inner and 80 for the outer detector are used to readout signals. Each channel uses three different gains 1, 1/7 and $1 / 49$ that provides the overall dynamic range of 0.2 to 2500 $\mathrm{pC}$, that is 5 times wider than the old ATM system. The width of the charge integration is $400 \mathrm{~ns}$ through a self-triggering scheme. Single photon resolution is $10 \%$ and $0.3 \mathrm{~ns}$, which is better than the intrinsic resolution of PMTs. The threshold is $-0.3 \mathrm{mV}$ corresponding to about 0.1 pe.

With this new electronics system, Super-K acquires a few new features. The individual neutrino events in a neutrino burst from supernovae can detect up to 6 million events for the first $10 \mathrm{~s}$ without any loss that is 100 times better than the previous Super-K phases. The detection efficiency for the $\mu \rightarrow e$ decays reaches about $100 \%$ for the first $1 \mu \mathrm{s}$. The detection of $2.2 \mathrm{MeV} \gamma$ after neutron capture becomes possible. These capabilities were impossible in the previous system.

Charged particles created by neutrino interactions in the water emit Cherenkov light. The opening angle of the Cherenkov light, $\cos \theta_{c}=1 / \mathrm{n} \beta$, is $42^{\circ}$ in water $(\mathrm{n}=1.33$ ) for relativistic particles. The Cherenkov threshold is 0.569 $\mathrm{MeV} / \mathrm{c}$ for electrons, $115.7 \mathrm{MeV} / \mathrm{c}$ for muons and $1.04 \mathrm{GeV} / \mathrm{c}$ for protons. The energy of the recoil charged particles can be obtained from the number of the observed photons. The number of photons from the Cherenkov radiation for unit path length is $\frac{d^{2} N}{d x d \lambda}=\frac{2 \pi \alpha Z^{2}}{\lambda^{2}}\left(1-\frac{1}{n^{2} \beta^{2}}\right)=\frac{2 \pi \alpha Z^{2} \sin ^{2} \theta_{c}}{\lambda^{2}}$.

The total photo-coverage was $40 \%$ except for the period of SK-II which was $20 \%$. By considering the Cherenkov photons produced, the PMT quantum efficiency and the averaged absorption of the photons, the $40 \%$ photo-coverage of the inner surface provides $\sim 6$ photo-electrons per $\mathrm{MeV}$. Threshold energy was initially $6 \mathrm{MeV}$, but soon decreased to 4.5 MeV in 1997 and kept at 4.5 MeV till 2008 except for the period of SK-II (2002-2005) where the number of inner PMT was reduced to about half. By making efforts to reduce the background in low energy, especially Rn in water, Super$\mathrm{K}$ has succeeded in lowering its energy threshold. Currently we are operating the detector with $3.0 \mathrm{MeV}$ threshold and the analysis threshold is $3.5 \mathrm{MeV}$. Although the new electronics record every pulse, the current energy threshold of $3.5 \mathrm{MeV}$ (kinetic energy) is limited by the background level. The event rate is $1.7 \mathrm{kHz}$ and $15 \mathrm{~Hz}$ of data above the software trigger threshold are recorded.

The energy ranges of the detectable neutrino events in Super-K are $3.5 \sim 15 \mathrm{MeV}$ ( (solar ${ }^{8} \mathrm{~B}$ neutrinos), $10 \sim 20$ $\mathrm{MeV}$ (neutrino burst from supernovae), $15 \sim 30 \mathrm{MeV}$ (relic neutrinos from past supernovae), $100 \mathrm{MeV} \sim$ a few $100 \mathrm{GeV}$ (atmospheric neutrinos), a few $100 \mathrm{GeV}$ (neutrinos from the annihilation of dark matter) and so on. The energy resolution for the low energy solar and supernova is $14.2 \%$ at $10 \mathrm{MeV}$ and that of the atmospheric neutrinos of single ring $\mu$ events is $(1.7+0.7 \sqrt{E(G e V)}) \%$.

Directions may be kinematically calculated to be $<18^{\circ}$ for the solar neutrinos with $10 \mathrm{MeV}$ through the $v_{x}+e \rightarrow$ $v_{x}+e$ interaction. But in reality the multiple scattering of electron in water limits the angular resolution to about $20^{\circ}$. For the high energy $v_{\mu}$ interactions $(>\mathrm{GeV}), v_{\mu}+X \rightarrow$ 
$\mu+X^{\prime}$, the direction can be determined in about $30^{\circ}$ for 1 $\mathrm{GeV}$ and $2^{\circ}$ for upward going $\mu$.

A water Cherenkov detector can determine in principle the energy and the direction of the recoil particles produced through the neutrino interactions as seen above, and the time with an accuracy of nano-second. These three measured quantities are also the basic quantities to do astrophysics in some cases.

\section{Neutrino oscillations}

The tiny neutrino masses and mixings are the ingredients to describe neutrino oscillations [12,13] and imply a physics beyond the standard model of elementary particle physics. For three active neutrinos ignoring sterile neutrinos, three mixing angles, three mass differences and one CP phase are needed. The Majorana phase is irrelevant for the oscillations. The mixing matrix is customary written [14] as

$$
\begin{aligned}
U_{\alpha l}= & \left(\begin{array}{ccc}
1 & 0 & 0 \\
0 & \cos \theta_{23} & \sin \theta_{23} \\
0-\sin \theta_{23} & \cos \theta_{23}
\end{array}\right)\left(\begin{array}{ccc}
\cos \theta_{13} & 0 & \sin \theta_{13} e^{-i \delta} \\
0 & 1 & 0 \\
-\sin \theta_{13} e^{i \delta} & 0 & \cos \theta_{13}
\end{array}\right) \\
& \left(\begin{array}{ccc}
\cos \theta_{12} & \sin \theta_{12} & 0 \\
-\sin \theta_{12} & \cos \theta_{12} & 0 \\
0 & 0 & 1
\end{array}\right) .
\end{aligned}
$$

The $\theta_{12}$ mixing is responsible for the solar neutrino oscillations and relevant to long baseline reactor neutrino oscillation experiments. The current best value is $\sin ^{2} \theta_{12}=$ $0.307 \pm 0.013$ [15]. The $\theta_{23}$ induces atmospheric neutrino oscillations and is measured by the accelerator long baseline neutrino oscillation experiments. The effect of the $\theta_{13}$ can be seen as a subdominant $v_{e}$ appearance effect in the atmospheric neutrinos and the accelerator neutrino oscillation experiments. Reactor experiments can extract the $\theta_{13}$ effect directly. The $\theta_{13}$ is small, $\sin ^{2} \theta_{13}=0.0212 \pm 0.0008$, but just large enough to study $\mathrm{CP}$ phase. The corresponding values of the mass differences measured so far are $\Delta m_{12}^{2}=(7.53 \pm 0.18) \times 10^{-5} \mathrm{eV}^{2}$ and $\Delta m_{23}^{2} \cong \Delta m_{13}^{2}=$ $(2.51 \pm 0.05) \times 10^{-3} \mathrm{eV}^{2}$.

The time evolution of the flavor eigenstates is

$$
\begin{aligned}
\left|v_{\alpha}(t)\right\rangle & =\sum_{j} U_{\alpha j} e^{-i E_{j} t}\left|\nu_{j}(0)\right\rangle \\
& =\sum_{j} \sum_{\beta} U_{\alpha j} U_{j \beta}^{*} e^{-i E_{j} t}\left|v_{\beta}(0)\right\rangle,
\end{aligned}
$$

$U^{*}$ denotes complex conjugate; $\alpha, \beta$ stand for flavor states, $\mathrm{e}, \mu, \tau$ and the $\mathrm{j}$ indices for the mass eigenstate $v_{1}, v_{2}, v_{3}$. Then the oscillation probability, $P\left(v_{\alpha} \rightarrow v_{\beta}\right)$ becomes

$$
P\left(v_{\alpha} \rightarrow v_{\beta}\right)=\left|\left\langle v_{\beta}(t) \mid v_{\alpha}(0)\right\rangle\right|^{2}
$$

$$
=\left|\sum_{j} U_{\beta j} U_{j \alpha}^{*} e^{-i E_{j} t}\right|^{2}
$$

We now know that we are in the fortunate situation that the mixing angle $\theta_{13}$ is very small. This smallness of the $\theta_{13}$ and the hierarchical structure of the neutrino masses, to first approximation, results in an effective decoupling. Therefore, the solar and the atmospheric neutrino oscillations can essentially be considered, separately. The early studies on the neutrino oscillation were indeed based upon the two neutrino oscillation scheme. The transition probability in the two flavor oscillation scheme, $v_{\alpha} \rightarrow v_{\beta}$ is

$P\left(v_{\alpha} \rightarrow v_{\beta}\right)=\sin ^{2} 2 \theta \sin ^{2}\left(\frac{1.27 \Delta m^{2} L}{E_{v}}\right)$,

where $\theta$ is the two flavor mixing angle, $\mathrm{U}=\left(\begin{array}{r}\cos \theta \sin \theta \\ -\sin \theta \cos \theta\end{array}\right)$; $\Delta m^{2}\left(\mathrm{eV}^{2}\right)$ is the mass squared difference; $\mathrm{L}$ (in $\mathrm{km}$ or $\mathrm{m}$ ) is the distance to the detector and $\mathrm{E}_{v}$ (in $\mathrm{GeV}$ or $\mathrm{MeV}$ ) is the neutrino energy. The wave length of the oscillation is $\lambda=4 \pi E_{v} / \Delta m^{2}$ or $\lambda(k m)=2.5 E_{v}(G e V) / \Delta m^{2}\left(e V^{2}\right)$.

In more precise studies, the sub-dominant effects are relevant. Therefore nowadays the atmospheric neutrino oscillations are analyzed in three flavor scheme. Small corrections due to the solar terms and the earth's resonance effect through $\theta_{13}$ need to be included. The neutrino mass hierarchy and $\mathrm{CP}$ violating effects can be extracted from the 3 flavor analysis. A study on the CP phase is only possible in the three neutrino scheme. For the solar neutrino oscillation, those effects in matter are very large and are the dominant effect. Small effects from $\theta_{13}$ must also be included. The results that include those effects will be described in the relevant sections. The study of neutrino oscillations in Super-K is eventually sensitive to all the mixing angles, mass differences and a CP phase.

\section{Atmospheric neutrinos}

\section{Atmospheric neutrino flux}

The primary cosmic rays, mostly protons, interact with molecules of the atmosphere and produce pions and kaons. Neutrinos are created by the decay of $\pi / \mathrm{K} \rightarrow \mu+v_{\mu}$ and also by the subsequent decay of $\mu \rightarrow e+v_{\mu}+v_{e}$. In order to make an accurate prediction of the neutrino flux, it is necessary to understand well the primary cosmic ray spectrum, hadron interactions (mostly of protons and heliums on the atmospheric nuclei) and production of the secondaries and their decays.

The flux of the primary cosmic rays has been measured by many experiments [16-19]. The uncertainty of the measured flux was significantly reduced over the last 10 years. AMS-02 
[20] on ISS has provided the latest measurements. They have extended the primary proton measurement up to $1.8 \mathrm{TeV}$. We should note that the average neutrino energy $\left\langle E_{v}\right\rangle$ roughly equals $\sim 1 / 10 \times\left\langle E_{p}\right\rangle$. For example $\sim 10 \mathrm{GeV}$ protons are responsible for $\sim 1 \mathrm{GeV}$ neutrinos.

The primary cosmic rays entering the earth's atmosphere are affected by the solar activity and the earth's geomagnetic field. The 11 year solar activity cycle acts on the solar wind that drives back the low energy cosmic ray particles out of the solar sphere. The resulting effect on the cosmic ray flux is about a factor of $\sim 5$ for $1 \mathrm{GeV}$ and $\sim 10 \%$ for 10 $\mathrm{GeV}$. The geomagnetic rigidity cut-off is a shielding effect by the earth's magnetic field that affects the low energy cosmic rays having entered in the earth's magnetosphere. The lowest rigidity of the primary cosmic ray able to reach the earth's surface depends on the location on the earth and the arriving direction. Therefore the flux of the atmospheric neutrinos is a function of time and depends on location and direction. We need to calculate the site dependent neutrino flux for each experiment as a function of time.

The primary cosmic ray particles arrive on the earth almost uniformly, as a consequence, the incoming direction of the atmospheric neutrinos are also nearly uniform except for the east-west effect [21] due to the earth's magnetic fields. In the low energy limit where the muons produced in the atmosphere decay before reaching the surface of the earth, the flux ratio of muon neutrinos to electron neutrinos, $R=\left(v_{\mu}+\overline{v_{\mu}}\right) /\left(v_{e}+\overline{v_{e}}\right)$, is close to 2 . When the energy increases, $\mathrm{R}$ increases, since less muons decay before reaching the ground. The observed spectrum, the flux times cross sections, peaks around $1 \mathrm{GeV}$ and extend up to a few $100 \mathrm{GeV}$ with a reduced rate of a few events per year. The neutrino and antineutrino ratio is slightly higher than one.

The uncertainly of the absolute neutrino flux has been improved over the last several years to $\sim 10 \%(<10 \mathrm{GeV})$ and $\sim 30 \%(\sim 100 \mathrm{GeV})$. If we take the ratio of the flux, $R=\left(v_{\mu}+\overline{v_{\mu}}\right) /\left(v_{e}+\overline{v_{e}}\right)$, then, the uncertainty in R (flux) is $3 \%$ for $<5 \mathrm{GeV}$ and $15 \%$ for $\sim 100 \mathrm{GeV}$. In the early stage of the oscillation analysis the ratio was used to see the effect of the neutrino oscillation.

The zenith angle distribution, especially in the ratio of the upward and the downward going events does not depend on the absolute flux calculation and is expected to be up/down symmetric. A slight asymmetry in the zenith angle distribution can be seen in low energy, which is originating from the effect of the geomagnetic cut off. The distribution becomes fully symmetric above 2-3 GeV. Therefore a flux independent evidence of the neutrino oscillation results, if an asymmetry is seen in the distribution. The uncertainty in the up/down ratio is estimated to be $1 \sim 2 \%$ for the energy below $5 \mathrm{GeV}$.

The angular correlation between incoming neutrinos and the corresponding outgoing leptons is poor below $\sim 500$
$\mathrm{MeV}$. The correlation becomes better for the energy above $500 \mathrm{MeV}$ to be less than $30^{\circ}$. Obviously the higher the energy, the better the angular correlation.

Neutrinos approaching the detector by crossing the earth interact in the rock beneath the detector and may produce high energy muons. Those muons entering the detector from the bottom are called upward going muons. Most of them cross and exit the detector. These upward going muons are also a direct signature of neutrinos. The muons produced in the atmosphere of the other side of the earth are eliminated in passing through the earth. This kind of events increases the sensitivity towards high energy, since the cross section $\sigma(v N)$ is proportional to $E_{v}$ and in addition to that the muon range is also proportional to $E_{\mu}$. The muon direction reflects the incoming neutrino direction within $2^{\circ}$. The uncertainty in the ratio of horizontal going and upward going muons is $\sim 2 \%$ mostly stemming from the uncertainty of the $\pi / K$ ratio.

Note that recently we were able to determine the unoscillated atmospheric neutrino flux from the measured atmospheric neutrino data since the neutrino oscillation parameters are now precisely known from the studies of many experiments [22].

\section{Atmospheric neutrino interaction in the detector}

The atmospheric neutrinos with energy of $\mathrm{O}(1 \mathrm{GeV})$ interacting in water produce leptons and are in some cases accompanied by hadrons. The charged current quasi-elastic (QE) interactions, $v+N \rightarrow l+N^{\prime}$, dominate below $1 \sim 2 \mathrm{GeV}$ and produce single ring events in a water Cherenkov detector. The charged current non-QE interactions comprise, single $\pi / K$ and multiple $\pi / K$ production and deep inelastic scattering (DIS), $v+N \rightarrow l+N^{\prime} s+\pi / K^{\prime} s$. Those processes form single or multi-Ring events are the backgrounds for the $\mathrm{QE}$ events. The cross section of the neutral current, $v+N \rightarrow v+N+\pi / K^{\prime} s$ is $\sim 1 / 3$ of the charged current interactions and create single ring and multi-ring events in Super-K. But the elastic neutral current scattering is not observable in water Cherenkov detectors. Super-K has about $40 \%$ detection efficiency for the total neutral current interaction. The latest parameters used in our simulation [23] were obtained by the front detectors of the T2K experiment [24]. The axial mass of the dipole form factor of the $\mathrm{QE}$ interaction is $\mathrm{M}_{A}[\mathrm{QE}]=1.137 \pm 0.034\left(\mathrm{GeV} / \mathrm{c}^{2}\right)$ and that of the resonance production is $\mathrm{M}_{A}$ [Res] $=0.724 \pm 0.052\left(\mathrm{GeV} / \mathrm{c}^{2}\right)$. The atmospheric neutrino events in Super-K are classified into 4 different event types:

- Fully contained events (FC): their event vertices are in the detector fiducial volume and all the particles produced are contained in the inner detector.

- Partially contained events (PC): their event vertices are in the detector fiducial volume and some particles exit from the inner detector. 
Super Kamiokande IV 1294.7 days : Monitoring

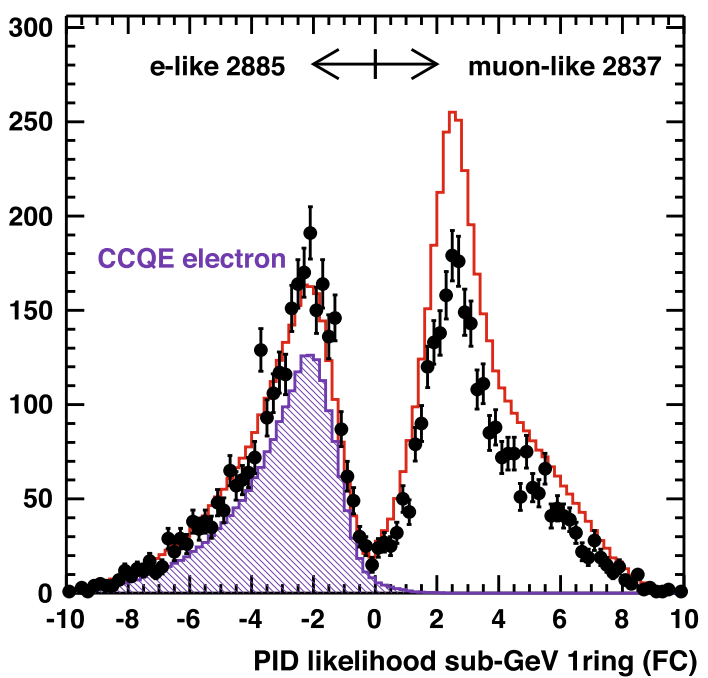

Fig. 3 The likelihood distribution of the sub-GeV events to separate $\mu$-like events from e-like events. The red line shows the MC calculation normalized to the e-like events. The lightly painted region represents the MC CCQE electron events. The mis-identification is less than $1 \%$ for sub-GeV and $\sim 2 \%$ for multi-GeV

- Upward going muons $(\mathrm{Up}-\mu)$ : entering the detector from the bottom of the detector.

- Upward stopping muons (Stop- $\mu$ ): Upward going muons, but stop in the inner detector.

The FC events are further divided into sub-GeV $\left(\mathrm{E}_{v i s}<1.33\right.$ $\mathrm{GeV})$ and multi-GeV $\left(\mathrm{E}_{v i s}>1.33 \mathrm{GeV}\right)$.

Data were processed through the following data reduction steps: (1) ring counting to categorize events to $1 \mathrm{R}$ (single ring), $2 \mathrm{R}$ (2 rings) and so on., (2) particle identification (ID) to classify each Cherenkov ring into $\mu, \mathrm{e} / \gamma$, proton and $\pi$ (still working on this $\pi$ identification), (3) vertex and energy momentum reconstruction, (4) fiducial volume cut ( $>2 \mathrm{~m}$ from the wall), (5) minimum energy cut of $>30 \mathrm{MeV}$ for $\mathrm{FC}$ and $\gtrsim 350 \mathrm{MeV}$ for PC events. For the energy reconstruction of electrons or muons, the observed total photo-electrons were used although there were many corrections.

About $70 \%$ of the total FC events are single ring events and we are able to separate events with up to 4 or 5 rings. We have used likelihood methods to separate $\mu$ 's and $\mathrm{e} / \gamma$ by an algorithm based on the diffuseness of the edge of the Cherenkov rings as shown in Fig. 3. The mis-identification probability is $0.6 \pm 0.1 \%$ for sub-GeV sample and $\sim 2 \%$ for multi-GeV. The ability of the particle identification was checked by using the cosmic ray $\mu$ and decay electrons, and the $\mathrm{e} / \mu$ test beam at KEK accelerator [25].

The fiducial mass for FC and PC is $22.5 \mathrm{kton}$ and the effective area for the Up- $\mu$ events is $\sim 1200 \mathrm{~m}^{2}$ where we require the minimum track length of the upward-going muons to be $1.7 \mathrm{~m}(1.6 \mathrm{GeV})$. The event rate is 8.2 events/day for
FC and 0.58 events/day for PC. The total number of events accumulated since the beginning of the experiment is listed in Table 1

Atmospheric neutrino events in Super-K cover a wide range of path lengths, i.e. three orders of magnitude, from $\mathrm{L} \sim 10$ (from the atmosphere above) to $\sim 13,000 \mathrm{~km}$ (crossing the earth) and a wide range of energy, $\mathrm{E}=\sim 0.1 \sim$ $10,000 \mathrm{GeV}$, five orders of magnitude. It is suited to explore searches for new phenomena in this wide range of coverage as well as to perform precise measurements.

\section{Atmospheric neutrino experiments prior to Super-K}

Atmospheric neutrinos were the background in the proton decay search that was the main objective of the Kamiokande experiment. In 1988, an anomaly in the atmospheric neutrino flux was revealed by the Kamiokande experiment that the double ratio $\left(\left(v_{e}+\bar{v}_{e}\right) /\left(v_{\mu}+\bar{v}_{\mu}\right)\right)_{\text {Data }} /\left(\left(v_{e}+\bar{v}_{e}\right) /\left(v_{\mu}+\right.\right.$ $\left.\left.\bar{v}_{\mu}\right)\right)_{M C}$, where $\left(v_{e}+\bar{v}_{e}\right)$ stands for e-like events and $\left(v_{\mu}+\bar{v}_{\mu}\right)$ stands for $\mu$-like events in the water Cherenkov detector, was smaller than 1 , about 0.6 [26]. This observation indicated either muon neutrinos were missing or electron neutrinos were in excess. It was addressed already in the past [27] that a neutrino oscillation may cause a deficit of atmospheric neutrinos. However, there were also skeptical views on the interpretation as a neutrino oscillation. For example there were concerns about the uncertainty of the atmospheric neutrino flux calculations, the validity of the neutrino interactions and so on. The effect of the polarization of muons that was not considered in the decay process in the earlier atmospheric neutrino calculation was also a concern. It was also not widely accepted by theorists that neutrinos may oscillate with large mixing.

It should be noted that there were also some experiments consistent with no deficits. Among those were NUSEX (150 tons) [28], Frejus (700 tons) [29], and Soudan-II (960 tons) [30]. All these experiments used Fe calorimeter techniques. The IMB detector using water Cherenkov technology same as Kamiokande, initially showed no deficit, but in 1992 paper [32], using sub-GeV data, they showed results consistent with Kamiokande by using the $\mu / \mathrm{e}$ separation technique. It was argued that there may be different systematics between water Cherenkov and calorimeter technology. But in 1997, finally the Sudan-II experiment [31] with larger statistics, confirmed the atmospheric neutrino anomaly. It was argued that the Frejus and NUSEX results suffered from small statistics.

In 1994, Kamiokande published the zenith angle distribution [33] with some indication of an asymmetry. However, the statistics was small and not conclusive. Therefore, it was commonly understood that it was important to make a precise measurement of the zenith angle distribution with higher statistics by Super-K.

The long baseline experiment, $\mathrm{K} 2 \mathrm{~K}$, has been planned already during the construction time of Super-K. The first 
neutrino beam derived from the KEK proton synchrotron was planned to be sent to Super-K in November, 1999.

\section{The discovery of neutrino oscillation in 1998}

The two flavor oscillation scheme was used for the atmospheric neutrino analysis at the early stage. This approach turned out to be practically correct due to some lucky situations. Since the mass difference of $\Delta m_{23}$ and $\Delta m_{13}$ is very close and then the oscillation between $v_{\mu} \rightarrow v_{\tau}$ through $\theta_{23}$ and $v_{\mu} \rightarrow v_{e}$ through $\theta_{23}$ and $\theta_{13}$ might mix. It is now known that $\theta_{13}$ is small, then the oscillation $v_{\mu} \rightarrow v_{e}$ through $\theta_{23}$ and $\theta_{13}$ cause nearly negligible effect especially when the experimental statistics was not sufficient to notice the effect from $\theta_{13}$ in their early stage.

Another accidental benefit came from the so called cancellation effect. In the low energy limit as described in the previous section, the flux ratio of $v_{e}$ to $v_{\mu}$ becomes approximately 2. Two mass differences $\Delta m_{23}$ and $\Delta m_{12}$ governing the atmospheric and solar neutrino oscillations are different by about one order of magnitude. Since the energy range of the atmospheric neutrinos is very wide, the atmospheric neutrino oscillation through $\theta_{12}$ can also be seen in the energy region of around $\leq 100 \mathrm{MeV}$. In this energy region, the oscillation length of $v_{\mu} \rightarrow v_{\tau}$ is much shorter than $v_{\mu} \rightarrow v_{e}$. Therefore the $v_{\mu}$ component is averaged out to $1 / 2$ before the $v_{\mu} \rightarrow v_{e}$ oscillation becomes visible due to the frequent oscillation between $v_{\mu} \leftrightarrow v_{\tau}$. So the initial flux ratio of $v_{\mu} / v_{e}=2$ in this energy region becomes effectively 1 , therefore $v_{\mu} \leftrightarrow v_{e}$ oscillation does not give a visible effect.

Due to the two situations mentioned above, the oscillation effect of atmospheric neutrinos was seen in $v_{\mu} \rightarrow v_{\tau}$, but not in $v_{\mu} \rightarrow v_{e}$. Also the size of the earth is just right to see the effect of up/down asymmetry of the $v_{\mu} \rightarrow v_{\tau}$ oscillations. Because of these lucky circumstances (of course we know that after the fact), we were able to obtain clear oscillation evidence consistent with the two flavor oscillation.

In Fig. 4, the zenith angle distributions for the e-like and $\mu$-like events in the multi-GeV region are shown. In the 535 days of data, asymmetries in the zenith angle distributions of the atmospheric $v_{\mu}$ are seen. The zenith angle represents a $L$ dependence of the event rate of the atmospheric neutrinos and therefore it gives direct evidence of the neutrino oscillation. Since the up/down asymmetry is less dependent of the flux calculations, this is compelling evidence for neutrino oscillations [34]. The data is consistent with the $v_{\mu} \rightarrow v_{\tau}$ oscillation. The best fit to the oscillation was $\chi_{\text {min }}^{2}=65.2 / 67 \mathrm{dof}\left(\sin ^{2} 2 \theta=1.0, \Delta m^{2}=2.2 \times 10^{-3} e V^{2}\right)$ while $\chi_{\min }^{2}=135 / 69$ dof was obtained for no oscillations. The significance of the deficits is $\Delta \chi^{2}=69.8$. The up/down ratio, $\sim 1-(1 / 2) \sin ^{2} 2 \theta$ becomes $\sim 1 / 2$ for full mixing for pure $v_{\mu}$ sample. The transition region depends upon $\Delta m^{2}$.
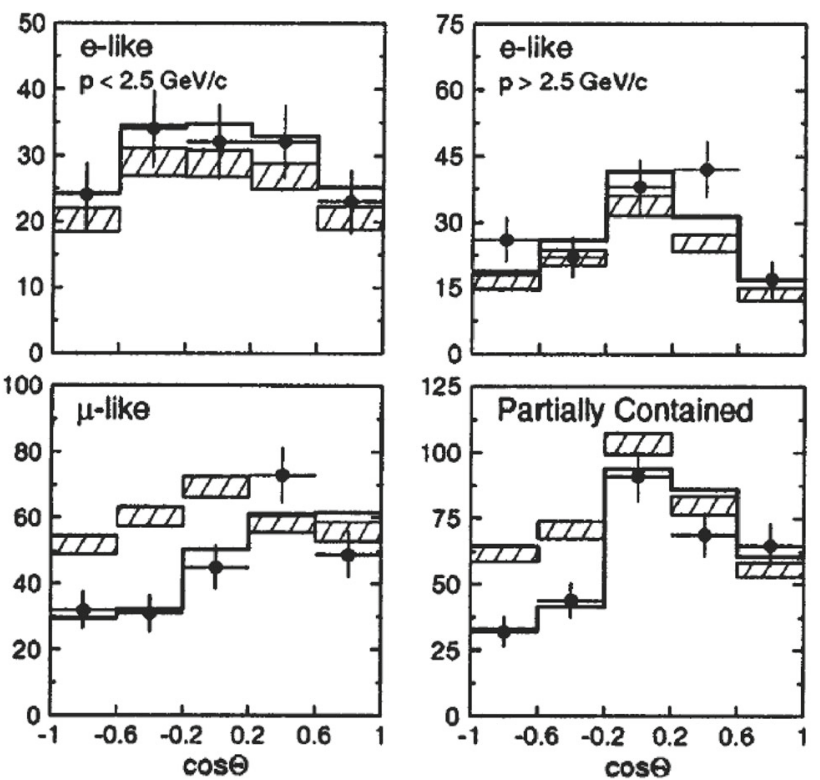

Fig. 4 Zenith angle distribution of the multi-GeV FC e-like, FC $\mu$-like and the partially contained events from 535 days of Super-K data (33.0 kt year) [34]. The vertical axis shows the number of events. The hatched data shows the events predicted without the oscillation. The fitted line for the best oscillation parameters are also shown. A clear deficit of upward going events $(\cos \theta \sim-1)$ in FC $\mu$-like and partially contained events is seen. This is the evidence of the atmospheric neutrino oscillation

The results about the evidence of neutrino oscillations were presented at XVIII International Conference on neutrino Physics and Astrophysics (NEUTRINO98) in June 1998 at Takayama Japan.

Before the Takayama conference, we had already made presentations at conferences about the results of the deficit of $v_{\mu} / v_{e}$ confirmed by the high statistics Super-K sub-GeV [35] and multi-GeV [36] data. We waited to have all the subsets of the data in order to give a consistent result before the announcement of the evidence of the neutrino oscillation. We waited in particular for the results from upward going muon data [37]. Just before the conference, everything was ready for the official announcement of the discovery of neutrino oscillations.

\section{Current situation of the atmospheric neutrinos}

It should be noted that the oscillation analysis has been improved and became precise as the data statistics increased over the last 20 years since the discovery [38-40]. With the higher statistics, the acquired events were further categorized into 19 sub-samples in order to enhance the sensitivity. Note that the number of sub-samples did vary as the experiment progressed. They were classified by the $v$ flavors (particle identification), event topologies (\# of rings), energies, number of decay electrons and so on. The zenith and momentum 

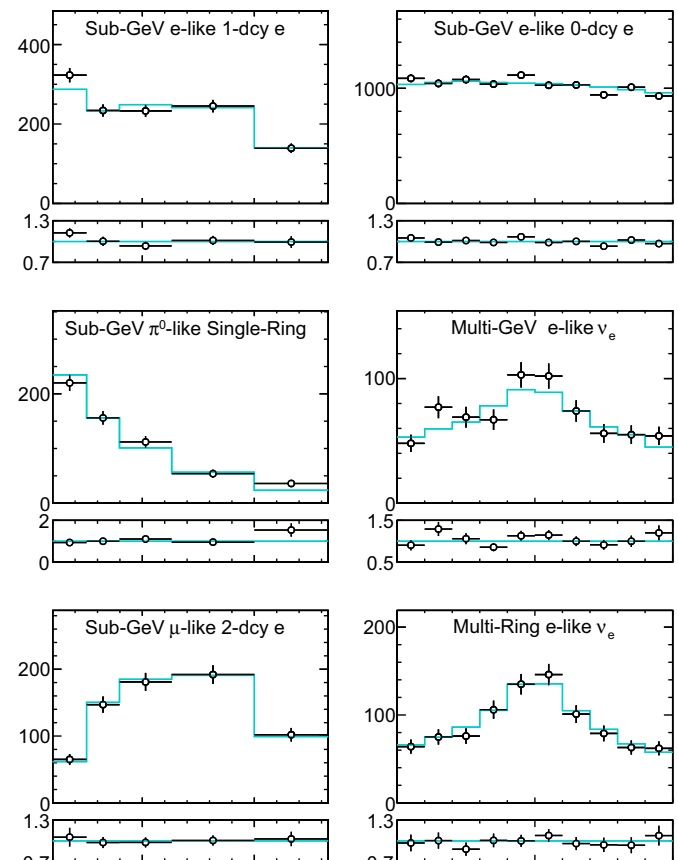

0.7
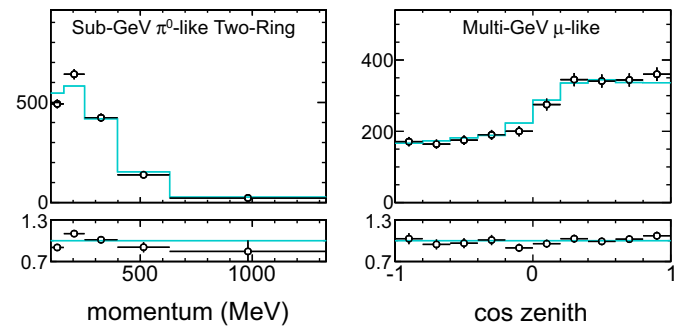
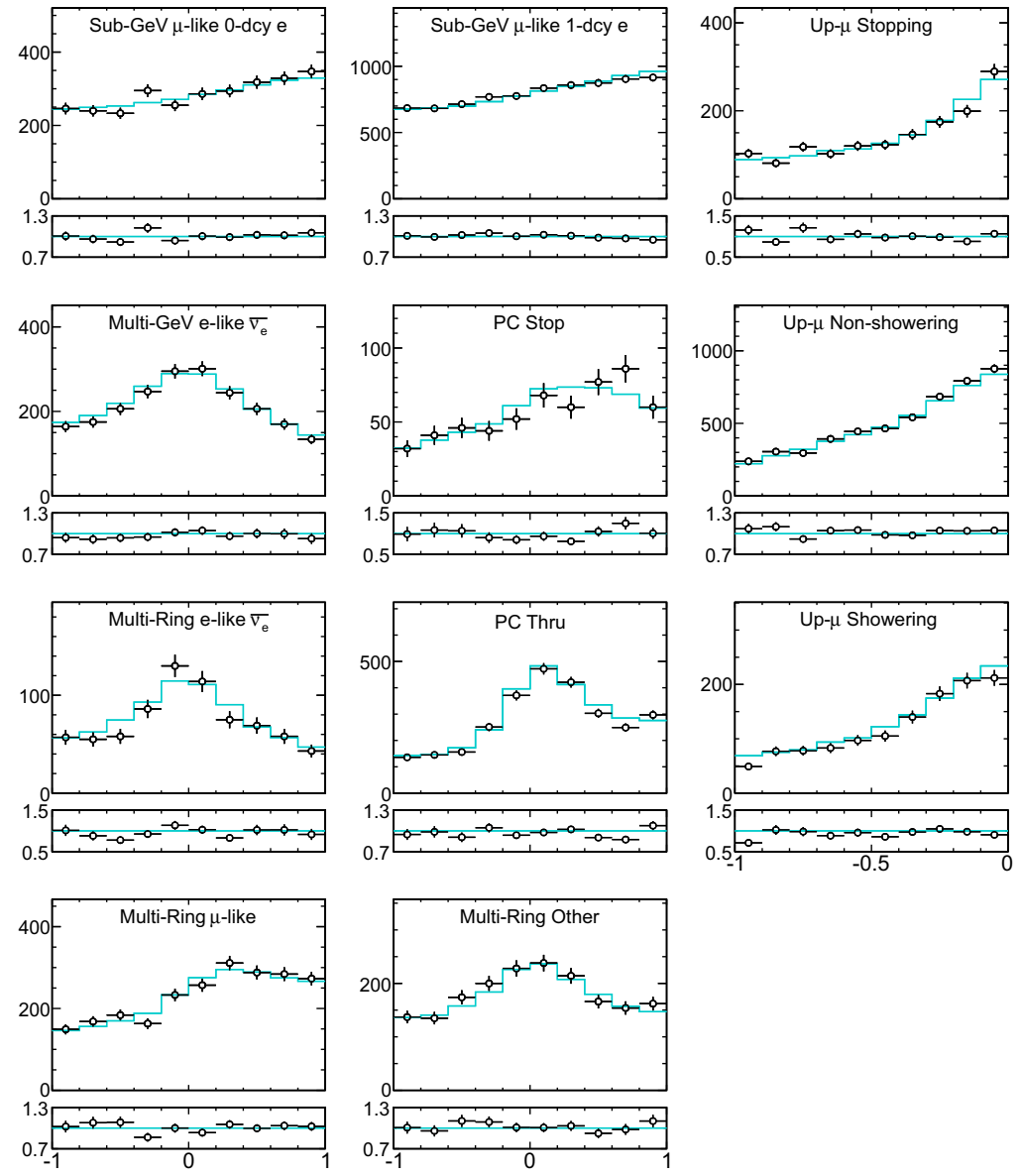

cos zenith

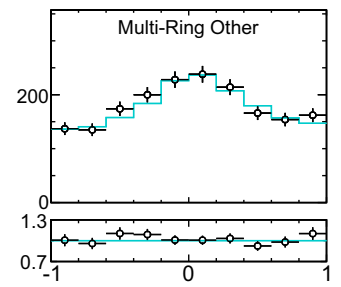

cos zenith

Fig. 5 Latest results of the atmospheric neutrino measurement [41]. Total $328 \mathrm{kton}$ - years of data spanning over SK-I to SK-IV are used for the fit. The histograms show the best fit of the MC. The sub-panels beneath each figure denotes the ratio with respect to the best fit

distributions of those sub-samples were the key data sets for the fitting. The data of each Super-K period was treated separately. The fit was performed over 520 analysis bins for each Super-K period and a total of 155 systematic error sources. The best oscillation fit to the data is shown in Fig. 5. The details of the analysis can be found in [41].

The sub-dominant contributions in the three flavor oscillation analysis from $\theta_{13}$, octant of $\theta_{23}$, mass hierarchy and CP phase can be seen, especially in $v_{e}$ appearance samples. The $v_{e}$ flux, $\Phi\left(v_{e}\right)$ as a consequence of the oscillation can be written [42],

$$
\begin{aligned}
& \frac{\Phi\left(v_{e}\right)}{\Phi_{0}\left(v_{e}\right)}-1 \approx P_{2}\left(r \cdot \cos ^{2} \theta_{23}-1\right) \\
& \quad-r \cdot \sin \tilde{\theta}_{13} \cdot \cos ^{2} \tilde{\theta}_{13} \cdot \sin ^{2} \theta_{23}\left(\cos \delta_{C P} \cdot R_{2}-\sin \delta_{C P} \cdot I_{2}\right) \\
& \quad-2 \sin ^{2} \tilde{\theta}_{13}\left(1-r \cdot \sin ^{2} \theta_{23}\right) \\
& \quad-\sin ^{2} \tilde{\theta}_{13} P_{2}(r-2)+\sin ^{4} \tilde{\theta}_{13}\left(1-r \sin ^{2} \theta_{23}\right)\left(2-P_{2}\right),
\end{aligned}
$$

where $\mathrm{r}$ is a ratio of the original $v_{\mu}$ to $v_{e}$ fluxes $(\sim 2.04$ to 2.06 for sub-GeV), $\tilde{\theta}_{13} \approx \theta_{13}\left(1+\frac{2 E V}{\Delta m_{13}^{2}}\right)$ (mixing angle in matter), $\mathrm{V}=\sqrt{2} G_{F} N_{e}, P_{2}=\left|\tilde{A}_{e \mu}\right|^{2}$ (amplitude of $v_{\mu} \rightarrow v_{e}$ in matter $), R_{2}=\operatorname{Re}\left(\tilde{A}_{e e}^{*} \tilde{A}_{e \mu}^{*}\right), I_{2}=\operatorname{Im}\left(\tilde{A}_{e e}^{*} \tilde{A}_{e \mu}^{*}\right) . \Phi_{0}\left(v_{e}\right)$ is the $v_{e}$ flux without oscillations.

The first term is the so called solar term, proportional to $\mathrm{P}_{2}$ that is the amplitude of $v_{\mu} \rightarrow v_{e}$ in matter. The matter effect is maximum at the resonance energy,

$E_{r e s} \sim\left(\Delta m_{12}^{2} \cos 2 \theta_{12}\right) /\left(2 V \cos ^{2} \theta_{13}\right)$.

For the current value of $\Delta m_{12}^{2}=7.6 \times 10^{-5} \mathrm{eV}^{2}$, and putting the known value of other mixing angles, the resonance is found to occur for $\mathrm{E} \lesssim 0.1 \mathrm{GeV}$. Therefore large matter effect can be seen in the low energy sample below 0.1 $\mathrm{GeV}$. Since $r=2.04 \sim 2.06$ for low energy, $r \cdot \cos ^{2} \theta_{23}-1$ becomes $0.02 \sim 0.03$. This is the cancellation effect already explained. Although this term is small, an excess of events can be seen for $\theta_{23}<45^{\circ}$ and a deficit can be seen for $\theta_{23}>45^{\circ}$. Therefore this term has a sensitivity to determine the octant $\left(\lessgtr 45^{\circ}\right)$ of $\theta_{23}$.

The second term is Ue3 term (see Sect. 3). The matter enhancement occurs at around $10 \mathrm{GeV}$ for $\Delta m_{13}^{2} \sim$ $2.3 \times 10^{-3} \mathrm{eV}^{2}$ causing a $5 \sim 10 \%$ effect. There is no can- 

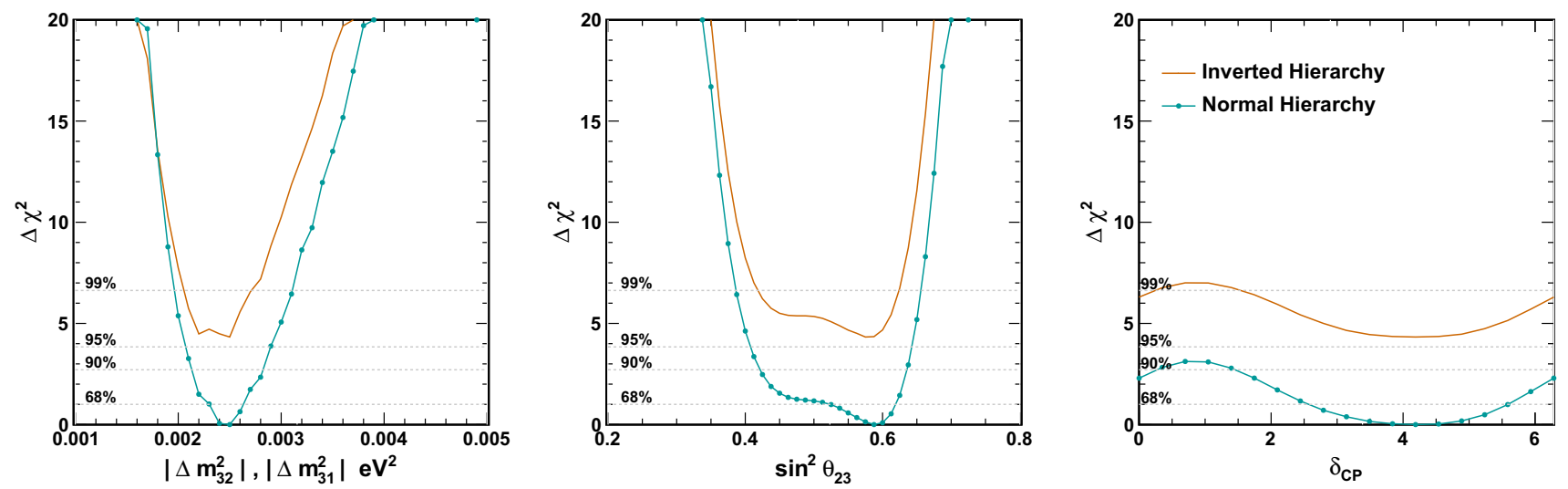

Fig. 6 Results of the fits of the Super-K atmospheric neutrino data, assuming $\sin ^{2} \theta_{13}=0.0219 \pm 0.0012$ [41]. Orange lines show the result for assuming the inverted hierarchy. Cyan lines denote the case for the normal mass hierarchy

cellation effect in high energy $(r>2)$. For the anti-neutrinos, $\bar{v}$, the matter potential changes its sign, $\mathrm{V} \leftrightarrow-\mathrm{V}$. Since in the resonance condition the potential is proportional to the mass difference, $\mathrm{V} \sim \Delta m^{2}$, therefore $v / \bar{v}$ undergoes the resonance for normal/inverted mass hierarchy case. The multi$\mathrm{GeV}$ samples are good to see the effect of $\theta_{13}$, mass hierarchy and $\mathrm{CP}$ phase at around $10 \mathrm{GeV}$. The effect is expected to be larger for the normal hierarchy than the inverted hierarchy. The sensitivity to the mass hierarchy strongly depends on $\theta_{23}$. If $\sin ^{2} \theta_{23}$ is larger than 0.55 , then the rejection capability of the wrong mass hierarchy becomes high and then the mass hierarchy may be determined in the very near future considering the current situation of the experimental results.

The third term is an interference term and depends on $\sin \tilde{\theta}_{13}$ linearly. This term is not strongly suppressed, but depends on the sign of $\sin \tilde{\theta}_{13}$ which is mass hierarchy dependent. There is no screening effect. It is also proportional to $\sin ^{2} \theta_{23}$ that means sensitive to the octant of $\theta_{23}$. The magnitude of the resonance effect depends upon whether the sensitivity to the $\mathrm{CP}$ phase, $\delta_{C P}$ is large or not.

The 4th term also stems from $\mathrm{U}_{e 3}$, but the contribution is negligible.

The latest atmospheric neutrino oscillation results of Super-K as of 2018 are shown in Fig. 6 where the value of $\theta_{13}$ was fixed at the best value from the reactor experiments including the uncertainty as a systematic error in the fit.

The results indicate that there is a weak preference of the normal mass hierarchy over the inverted hierarchy at 93\% assuming the best fit point. The constraints on the oscillation parameters by assuming the normal mass hierarchy are $\sin ^{2} \theta_{23}=0.588_{-0.064}^{+0.031}, \Delta m_{32}^{2}=2.50_{-0.20}^{+0.13}$ and $\delta_{C P}=4.18_{-1.61}^{+1.41}$.

As noted Super-K was used as far detector in the long baseline (LBL) neutrino oscillation experiments. This idea has been expanded and developed rapidly. In 2004, a new long baseline neutrino oscillation experiment, T2K, using high intense neutrino beam from JPARC had started in order to explore a neutrino oscillation through $U_{e 3}$, mass hierarchy and CP-phase. The long baseline experiments have become a major tool to study neutrino oscillations, which provides high statistics and well controlled neutrino data, suiting especially to explore tiny effects of mass hierarchy and CP phase. Surprisingly enough it was planned before the discovery of neutrino oscillations. The combined analyses with $\mathrm{T} 2 \mathrm{~K}$, where the Super-K detector was used as a far detector, definitely improved the results, but will not be discussed further, as it is outside the scope of this article.

\section{Solar Neutrinos}

\section{Solar neutrino flux}

The solar energy originates from nuclear fusion reactions taking place in its central core. The net reaction is

$4 \mathrm{p} \rightarrow{ }^{4} \mathrm{He}+2 \mathrm{e}^{+}+2 v_{e}+26.2 \mathrm{MeV}$.

Most of the energy is transferred to the kinetic energy of the charged particles and photons and will eventually be emanated from the surface of the sun $\left(3.9 \times 10^{33} \mathrm{erg} / \mathrm{s}\right.$ [solar luminosity]), several 10,000 years later. The neutrinos carry away only $\sim 3 \%$ of the generated energy, but they leave the surface of the sun in about $2 \mathrm{~s}$ after the creation at the core.

The pp-chain is the dominant process in the sun ignited at the relatively lower core temperature of $1.5 \times 10^{7} \mathrm{~K}^{\circ}$ [43]. There are five neutrino production processes in the pp-chain and those neutrinos from different processes are called by their specific names as listed in Table 2. There are small contributions from CNO cycles where hydrogen is burned using carbon as a catalyst where relatively low energy neutrinos around $1-2 \mathrm{MeV}$ are produced that Super-K cannot detect [44-46]. 
Table 2 Solar neutrinos [44]

\begin{tabular}{lllll}
\hline $\begin{array}{l}\text { Solar } \\
\text { neutrinos }\end{array}$ & $\begin{array}{l}\text { Production } \\
\text { interactions }\end{array}$ & $\begin{array}{l}\text { Q-value } \\
(\mathrm{MeV})\end{array}$ & $\begin{array}{l}\text { Line } \\
(\mathrm{MeV})\end{array}$ & $\begin{array}{l}\text { Flux } \\
\left(\times 10^{10} \mathrm{~cm}^{-2} \mathrm{~s}^{-1}\right)\end{array}$ \\
\hline $\mathrm{pp}-v$ & $\mathrm{p}+\mathrm{p} \rightarrow \mathrm{d}+\mathrm{e}^{+}+v_{e}$ & 0.42 & - & $6.03(1 \pm 0.006)$ \\
$\mathrm{pep}-\boldsymbol{v}$ & $\mathrm{p}+\mathrm{e}+\mathrm{p} \rightarrow \mathrm{d}+v_{e}$ & - & 1.2 & $0.0147(1 \pm 0.012)$ \\
${ }^{7} \mathrm{Be}-v$ & $\mathrm{e}+{ }^{7} \mathrm{Be} \rightarrow{ }^{7} \mathrm{Li}+v_{e}$ & - & $0.8,0.4$ & $0.456(1 \pm 0.07)$ \\
${ }^{8} \mathrm{~B}-\nu$ & ${ }^{8} \mathrm{~B} \rightarrow{ }^{8} \mathrm{~B}^{*}+\mathrm{e}^{+}+v_{e}$ & 15 & - & $4.59(1 \pm 0.14) \times 10^{-4}$ \\
hep- $v$ & ${ }^{3} \mathrm{He}+\mathrm{p} \rightarrow{ }^{4} \mathrm{He}+\mathrm{e}^{+}+v_{e}$ & 20 & - & $8.31(1 \pm 0.3) \times 10^{-7}$ \\
\hline
\end{tabular}

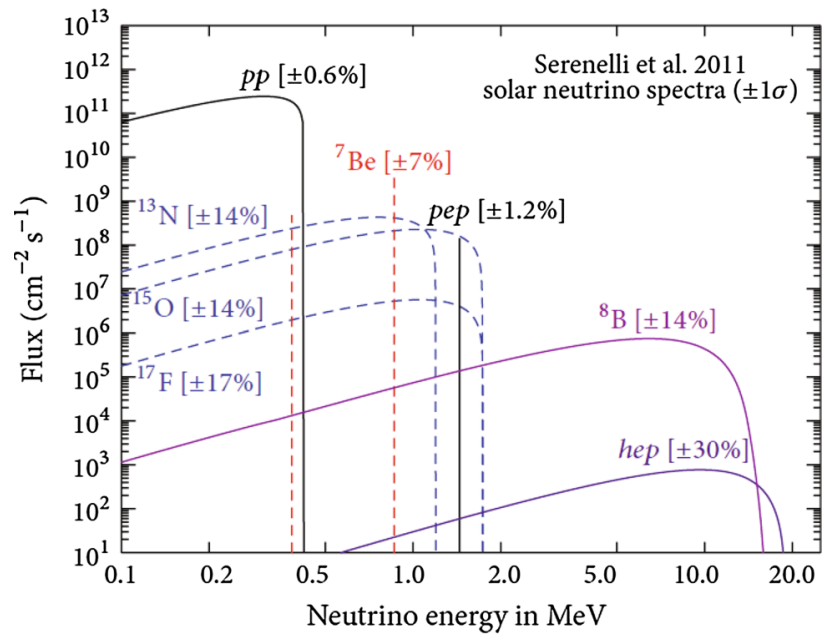

Fig. 7 Solar neutrino spectrum [47]. Super-K is able to measure only ${ }^{8} \mathrm{~B}$-solar neutrinos above $3.5 \mathrm{keV}$

It is easily obtained from the elementary process in the sun and the solar luminosity that the total solar neutrino flux is of $6.6 \times 10^{10} \mathrm{~cm}^{-2} \mathrm{~s}^{-1}$ at the top of the atmosphere of the earth. Individual fluxes of pp, pep, ${ }^{7} \mathrm{Be},{ }^{8} \mathrm{~B}$ and hep neutrinos calculated by the solar model [44] are also listed in Table 2 . The contributions from the $\mathrm{CNO}$ neutrinos are about $2 \%$ of the flux of the pp-neutrinos. The spectrum of the solar neutrinos is shown in Fig. 7.

\section{Solar neutrino problem}

The Homestake Chlorine experiment $[48,49]$ started in the late ' 60 s observed initially that the solar neutrino flux was significantly lower than expected. This was called the "solar neutrino problem or puzzle". The Chlorine experiment, i.e. a radio-chemical experiment, counts the number of ${ }^{37} \mathrm{Ar}$ atoms created through the solar neutrino interaction, the inverse beta decay of $v_{e}+{ }^{37} \mathrm{Cl} \rightarrow e^{-}+{ }^{37} \mathrm{Ar}$ with the energy threshold of $817 \mathrm{keV}$ and is sensitive mostly to ${ }^{8} \mathrm{~B}(\sim 75 \%)$ and ${ }^{7} \mathrm{Be}(\sim 15 \%)$ neutrinos. The experiment observed about $1 / 3$ of the flux predicted.

The Chlorine experiment was the only solar neutrino experiment for about 20 years till the late ' 80 s and the results were persistent during the periods. Possible interpretations of this deficit were (1) experimental problems (systematic errors), (2) astrophysical problems (incorrectness of the solar model) and (3) neutrino problems (oscillations).

We should note that the radio chemical experiments were not familiar to the physicists who had to admit an amazing chemical procedure to extract a few atoms out of a few hundred tons of material. It was also known that the predictions of the fluxes of ${ }^{7} \mathrm{Be}$ and ${ }^{8} \mathrm{~B}$ neutrinos that were responsible for the Chlorine measurement had large uncertainties. Especially the astrophysical S-factor, $\mathrm{S}(\mathrm{E})_{17}$, was not well known in '70s $\sim$ ' 80 s. In addition, the deficit of 1/3 could not accommodate a simple two flavor vacuum oscillation interpretation. The MSW effect [50,51], the resonance enhancement in the propagation of neutrinos in matter was first presented in 1985. It should be noted that this $1 / 3$ deficit is still a puzzle and not quite consistent with the finally chosen large mixing angle (LMA) solution. The last remark is that in addition to the deficit, the $\mathrm{Cl}$ experiment had claimed an anti-correlation of the flux of the solar neutrinos with the sunspot numbers, the 11-year solar activity. We now know that the anti-correlation was not confirmed by the later experiments, but had caused confusion.

For the $\sim 20$ years after the initial claim of the deficit, in light of the results from the new experiments, the possible explanations were gradually changing and converging on neutrino oscillations.

The second solar neutrino experiment, Kamiokande, had succeeded to observe solar neutrinos in 1989 [9] and made also a first measurement of the energy spectrum. It is a real time and a directional measurement through the $v+e \rightarrow v+e$ interaction with a threshold of $7 \mathrm{MeV}$. Kamiokande observed $55 \%$ of the expected flux and verified that those neutrinos were really coming from the sun. Kamiokande confirmed the Chlorine observation of the long-standing solar neutrino deficit and concluded that the deficit was not entirely an experimental problem and revealed that a further study on the precise flux calculation and the neutrino oscillation were needed. Note that the detection of ${ }^{8} \mathrm{~B}$ neutrinos was a proof of existence of the pp-chain in the sun.

In 1990, SAGE (Soviet American Gallium Experiment) [52] presented their first results. They used ${ }^{71} \mathrm{Ga}$ as a target material counting solar neutrinos through the interaction, 


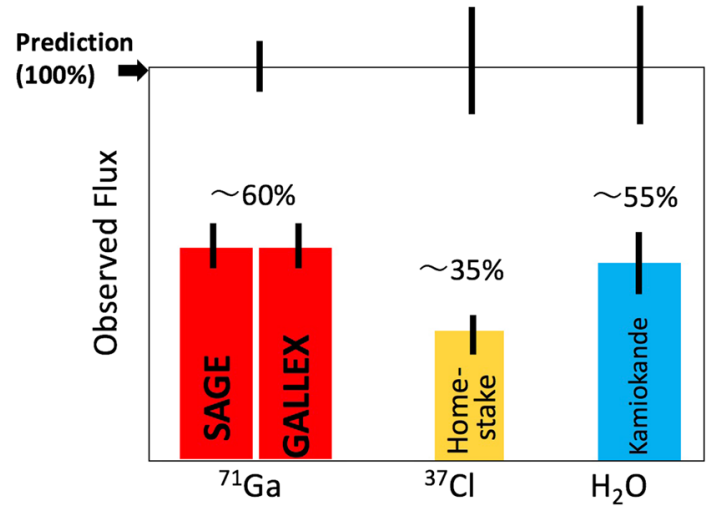

Fig. 8 Results from the 4 solar neutrino experiments in the middle of 90's. All the experiments show the flux deficits

$v_{e}+{ }^{71} G a \rightarrow e^{-}+{ }^{71} G e$, with the threshold energy of 250 $\mathrm{keV}$. For the radiochemical experiments, solar models can predict the share of "capture rate", the Ga experiments of which has $\sim 55 \%$ for pp-neutrinos, $\sim 25 \%$ for ${ }^{7}$ Be-neutrinos and $\sim 10 \%$ for ${ }^{8} \mathrm{~B}$-neutrinos. There is a strong constraint on the amount of the pp-neutrinos from the solar luminosity, with the conclusion that the prediction on the capture rate of the pp-neutrinos is very solid and the uncertainty is only $1 \sim 2 \%$.

In 1991, the GALLEX experiment [53,54], another Ga experiment, confirmed the solar neutrino deficits of about $55 \%$ of the predicted capture rate. The two experiments eventually provided consistent results. We should take in account two facts. The expected capture rate of 55\% from pp-neutrino has very small uncertainties due to the luminosity constraint. We know that ${ }^{7} \mathrm{Be}$ and ${ }^{8} \mathrm{~B}$ neutrinos exists as a consequence of the $\mathrm{Cl}$ and Kamiokande measurements which add an additional solid "capture rate" on top of the pp-neutrino capture rate. Therefore the observed deficit of $55 \%$, are not explained by the uncertainty of the solar models.

Those results on the flux deficit of the four different experiments had been persistent and became stronger during the time of the Super-Kamiokande construction in the early ' 90 s. The results of the four solar neutrino experiments are schematically shown in Fig. 8.

In the early ' 90 s, it became widely presumed that those deficits of solar neutrinos were caused by neutrino oscillations. But Super-Kamiokande could only measure high energy ${ }^{8} \mathrm{~B}$ neutrinos, leaving the flux uncertainty still as a concern. Therefore, we have conceived a new type of analysis in order to obtain definitive evidence of neutrino oscillations independent of the flux calculations. One is to look for a spectrum distortion and another one is to find a time dependence. Some oscillation parameters predict a spectrum distortion or a time variation of the flux.

\section{Solar neutrino oscillation study before Super-K}

In order to see the solar neutrino situation in early ' 90 s more clearly, we recollect the analysis done in those days. The results of the 4 solar neutrino experiments were analyzed assuming neutrino oscillations. When we handle the propagation of neutrinos through the sun, the MSW effect (the matter effect in the continuously changing density) $[50,51]$ must be taken into account. The mixing angle in matter, $\theta_{m}$, for the two neutrino case is written as,

$\tan ^{2} 2 \theta_{m}=\frac{\tan ^{2} 2 \theta_{V}}{1-\left(2 p \sqrt{2} G_{F} n_{e}\right) /\left(\delta m^{2} \cos 2 \theta_{V}\right)}$,

where $\delta m^{2}$ is the mass squared difference of $\left|m_{1}^{2}-m_{2}^{2}\right|$. The electron density in the matter is shown as $n_{e}$. And $\theta_{V}$ is the mixing angle in vacuum.

The matter effect has a resonance for

$1 \sim\left(2 p \sqrt{2} G_{F} n_{e}\right) /\left(\delta m^{2} \cos 2 \theta_{V}\right)$.

The resonance condition in the adiabatic transition for 10 $\mathrm{MeV}$ neutrinos was satisfied for $\delta m^{2} \leq 1.6 \times 10^{-4} \mathrm{eV}^{2}$. But the adiabatic condition breaks down at $\delta m^{2} \geq 6.3 \times$ $10^{-8} \cos ^{2} 2 \theta / \sin ^{2} 2 \theta \mathrm{eV}^{2}$ for $10 \mathrm{MeV}$ neutrinos.

Performing a fit including the MSW effect, four distinct regions in the $\left(\Delta m^{2}, \sin ^{2} 2 \theta\right)$-plane are allowed, they are called: the large mixing angle solutions, the small mixing angle solutions, LOW and the vacuum solutions as shown in Fig. 9. They have different energy dependent suppressions of the solar neutrino spectrum.

For the large mixing angle solution (we now know that this is the right solution), the low energy neutrinos like pp, pep and ${ }^{7} \mathrm{Be}$-neutrinos undergo vacuum oscillation, whereas the high energy neutrinos above a few $\mathrm{MeV}$ undergo matter conversions. In these energy regions which Super-K can cover, nearly the flat energy suppression is expected. Possible day/night flux differences in the higher energy regions are expected at the few \% level.

We expect strong spectrum suppression at the low energy side for the small mixing angle solutions. Nearly uniform suppression in the relevant energy region and large day/night flux difference in the low energy region are expected for the LOW solution. But the probability of the LOW solution to be the right solution is very low. We expect seasonal variations and spectrum distortions for the vacuum oscillations.

The possible four solutions above have the distinctive and desirable nature to be independent of the flux calculations like the spectrum distortions and the time variations (day/night and seasonal variations). A relevant experiment needs to accumulate enough statistics to explore those characteristics. Therefore the large size and the high sensitivity of Super-K is needed. 


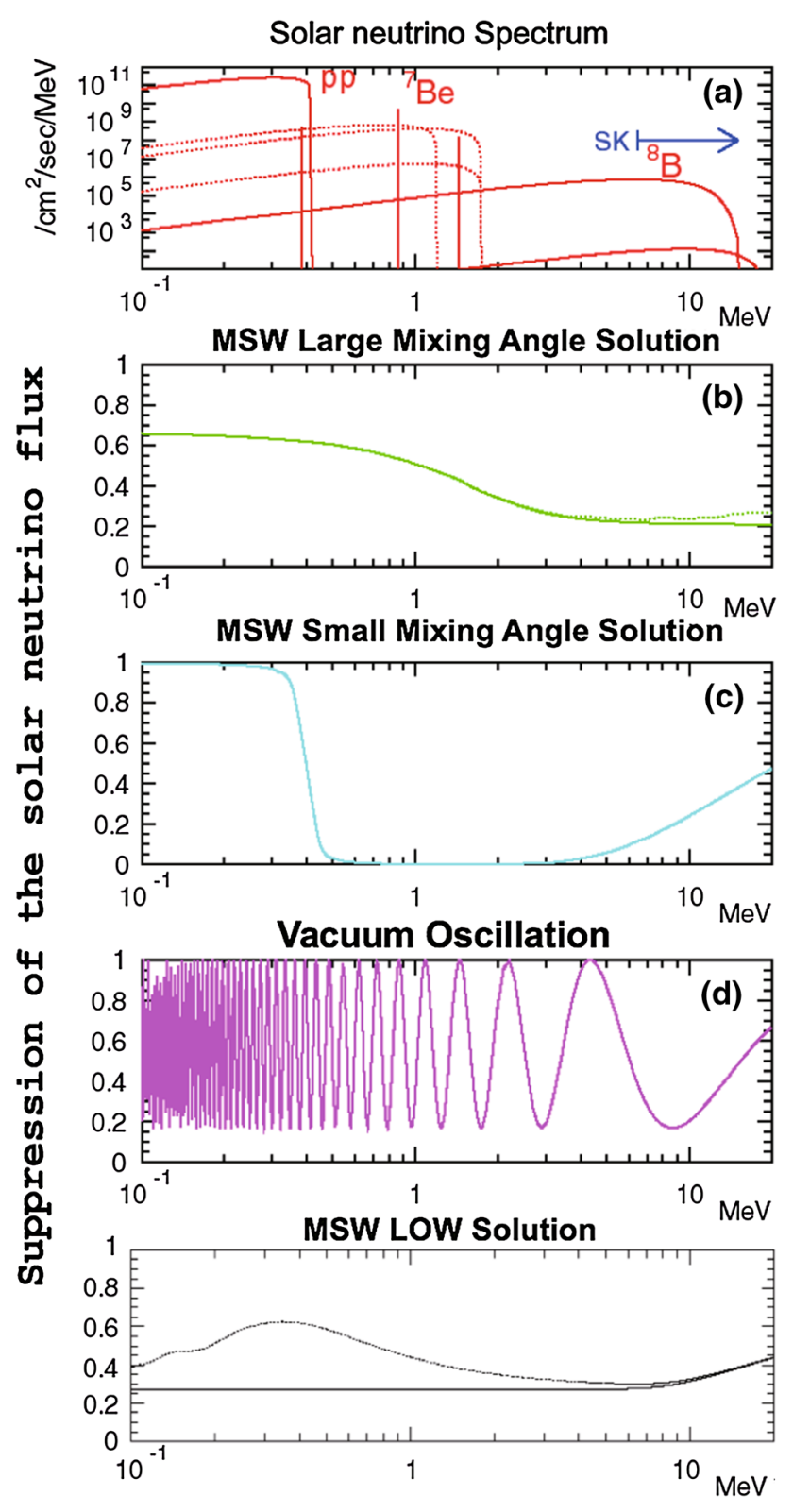

Fig. 9 The energy spectra of the four possible solutions. The small mixing angle solutions and the vacuum solutions show strong distortion of the energy spectrum and the large mixing angle solution gives a day/night flux difference. A seasonal flux difference can be seen for the vacuum solutions

\section{Super-K and solar neutrinos}

Low energy solar neutrinos mostly interact with electrons in the water Cherenkov detector. The electron neutrinos undergo charged and neutral current interactions whereas the oscillated $v_{\mu}$ and $v_{\tau}$ interact only through the neutral current. The differential cross section of the $v+\mathrm{e} \rightarrow v+\mathrm{e}$ interaction is given as

$$
\frac{\mathrm{d} \sigma}{\mathrm{d} T}=\frac{2 \mathrm{G}_{F} m_{\mathrm{e}}}{\pi}\left[g_{L}^{2}+g_{R}^{2}\left(1-T / E_{v}\right)^{2}-g_{L} g_{R} m_{e} T / E_{\nu}^{2}\right],
$$

where $\mathrm{T}$ is a kinetic energy of the recoil electron and the couplings are given by $g_{L}=1 / 2+\sin ^{2} \theta_{W}$ and $g_{R}=\sin ^{2} \theta_{W}$ for $v_{e} \mathrm{e}$ interactions. Practically $\sigma\left(v_{\mu,(\tau)} \mathrm{e}\right) / \sigma\left(v_{e} \mathrm{e}\right) \simeq 0.15$.

For the neutrinos of averaged energy of $10 \mathrm{MeV}$, interacting in the water Cherenkov detector, the direction of the recoil electrons keeps that of neutrinos within $\theta_{v e}^{2}<2 \mathrm{~m}_{e} / \mathrm{E}$. For example, the typical direction of $10 \mathrm{MeV}$ solar neutrinos is constrained kinematically to be less than 18.6 degree. However, due to the multiple scattering in water the angular resolution increases to $\sim 26^{\circ}$ for $10 \mathrm{MeV}$.

The vertex, the direction and the energy are well reconstructed for solar neutrino events around a few MeV. The number of photo-electrons (pe) observed is about $6 \mathrm{pe} / \mathrm{MeV}$ and therefore we have $30 \mathrm{PMT}$ hits for $5 \mathrm{MeV}$ recoil electron events. The vertex was reconstructed by using the PMT timing information. The maximum likelihood method making use of the Cherenkov ring pattern was used to obtain the direction of recoil electrons. Note that in addition to the regular calibration system using radioactive sources, an electron LINAC was arranged in situ to inject electrons with known energy at various positions inside of the water tank. We have performed this LINAC calibration twice per year.

The measurement of ${ }^{8} \mathrm{~B}$ neutrinos in low energy is limited by backgrounds. Most backgrounds came from the spallation products emanating from the preceding high energy muons, $\gamma$-rays from external origin and also ${ }^{222} \mathrm{Rn}$ contaminated in the water. In order to reduce the backgrounds, we have first applied cuts to eliminate noise events. We have basically selected isolated events more than $20 \mu \mathrm{sec}$ apart from the time adjacent events with clean and well recognized Cherenkov patterns. Correlations to the preceding muons are important information about the spallation products. Many of the spallation products are produced along the track of muons and the higher the energy of muons, the more the spallation products are produced. Making use of those correlations, $98 \%$ of the spallation products were removed while keeping the signal efficiency near $80 \%$. The inner 22.5 ktons of fiducial mass was used for the solar neutrino analysis and furthermore the incoming $\gamma$-rays were selectively removed by introducing a cut on the distance to the PMT wall along the backward event direction. In the past 20 years of experimenting the analysis algorithm has been improved, and cut parameters for the spallation products and the $\gamma$-rays were improved but their basic concepts remained unchanged as outlined in our different papers for the respective analyses in the respective time periods.

When we go down to the low energy region, we have added additional cuts to reduce the backgrounds further. The event rate with $5 \mathrm{MeV}$ energy threshold in the 22.5 ton fiducial mass is about 10 per day. In the latest analysis threshold level as small as $3.5 \mathrm{MeV}$ (K.E.) has been achieved and further efforts to reduce the threshold has been made. 
Most of the remaining backgrounds are the daughters of ${ }^{222} \mathrm{Rn} .{ }^{214} \mathrm{Bi}$ gives a high energy $\beta$-ray with the end point energy of $3.3 \mathrm{MeV}$ where the resolution tail mimic the solar neutrino interactions. The Rn contamination has been reduced after the many years of struggle and now become less than $0.1 \mathrm{mBq} / \mathrm{m}^{3}$.

\section{Discovery of the solar neutrino oscillation}

The solar neutrino study by Super-K had begun in April, 1996 aiming at obtaining the compelling and definitive evidence of the oscillation [55-57]. Unfortunately it took longer time than expected to find the solution to the solar neutrino problem and the conclusive result came from a different corner.

In 2001 the Super-K Collaboration published two papers, one of them showed the results of the precise measurement of the ${ }^{8} \mathrm{~B}$ solar neutrino flux using 1258 days of data [58]. The measured solar neutrino flux between 4.5 and $19.5 \mathrm{MeV}$ (recoil electron kinetic energy) was $2.32 \pm$ 0.03 (stat.) $)_{-0.07}^{+0.08}$ (syst.) $\times 10^{6} \mathrm{~cm}^{-2} \mathrm{~s}^{-1}$ that was $45.1 \pm$ 0.5 (stat.) ${ }_{-1.4}^{+1.6}$ (syst.) $\%$ of the predicted value of the standard solar model.

The other paper presented the results on searches for a deviation from the $\beta$-decay spectrum shape and for time variations of ${ }^{8} \mathrm{~B}$ solar neutrinos [59]. No energy spectrum distortion and no seasonal variations were found. Therefore the small mixing angle solutions and vacuum oscillations were rejected from the right answer as shown in Fig. 10. This exclusion was independent of the flux calculations of the standard solar models.

Those Super-K results, then, strongly indicated that the right answer is the large mixing angle solutions (LMA) as a consequence of the elimination of other possible solutions consisting in not detecting their characteristic flux independent signatures.

But LMA itself does not have a strong model independent characteristics. LMA shows a uniform spectrum suppression with no energy distortion and a small day/night flux difference. Super-K observed a day/night effect $(1.3 \sigma)$, but was not significant statistically to evidence LMA as a right solution. Note that 13 years later, Super-K obtained $3 \sigma$ effect on the day/night flux difference.

This was a strange situation. Though Super-K indicated that the allowed parameters were consistent with LMA, but the Super-K results were, however, not sufficient to demonstrate that LMA was the right answer for the solar neutrino problem.

In June 18th, 2001, when the above 2 papers from Super-K were published, SNO [60], the $1 \mathrm{kton}$ heavy water Cherenkov detector in Canada, announced the first result of their measurement on the charged current interactions, $v_{e}+d \rightarrow$

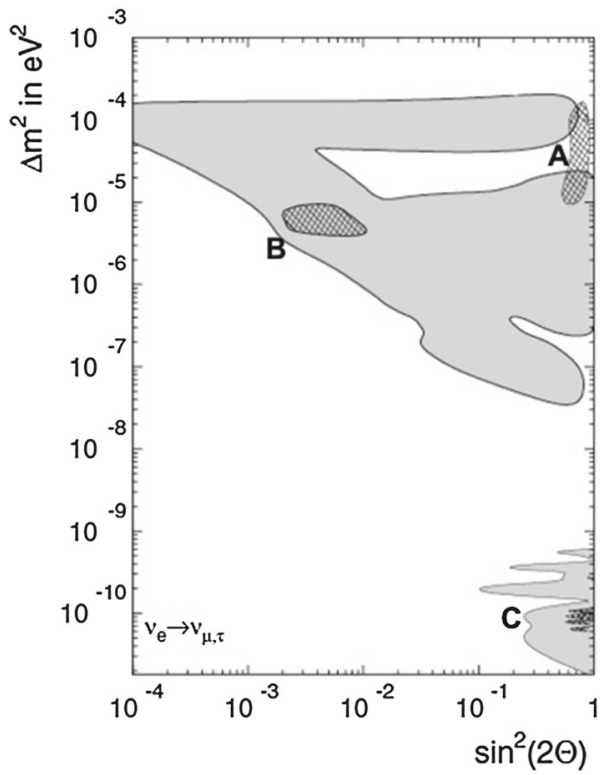

Fig. 10 Excluded region only by the Super-K energy spectrum and time variation measurement without the flux normalization are shown by the gray region [59]. The allowed parameter regions by all the solar neutrino experiments including the flux normalization are shown by the hatched regions: a the large mixing angle solutions (LMA); $\mathbf{b}$ the small mixing angle solutions (SMA); and $\mathbf{c}$ the vacuum oscillation solutions (VO). SMA and VO are excluded by Super-K. LMA remain as possible oscillation parameters

$p+p+e^{-}$, which was exclusively sensitive to $v_{e}$ 's. The $v_{e}$ flux observed by SNO was $1.75 \pm 0.07$ (stat. $)_{-0.11}^{+0.12}$ (syst.) \pm 0.05 (theor.) $\times 10^{6} \mathrm{~cm}^{-2} \mathrm{~s}^{-1}$.

In order to obtain definitive evidence of the flux suppression by solar neutrino oscillations, two separated measurements are necessary, namely the measurement of $v_{e}$ flux and a flux that includes information of $v_{v, \tau}$. Both the neutral current interactions and $v+e$ elastic scattering interactions are eligible for the second measurement.

The charged current result from SNO [60] and the neutrino electron scattering measurement from Super-K were compared. We quote from the abstract of SNO paper the statement "Comparison of $\phi^{C C}\left(v_{e}\right)$ to the Super-Kamiokande Collaboration's precision value of the flux inferred from the ES reaction yields a $3.3 \sigma$ difference, assuming the systematic uncertainties are normally distributed, providing evidence of an active non- $v_{e}$ component in the solar flux." This is the first evidence of the solar neutrino as shown in Fig. 11 changing to other neutrinos during the travel from the sun to the earth. It is quite interesting that the discovery of the solar neutrino oscillation was achieved by studying only $1 / 10,000$ of the tail of the solar neutrino flux. 
$\phi\left(v_{\mathrm{e}}\right)$ (relative to BPB01)

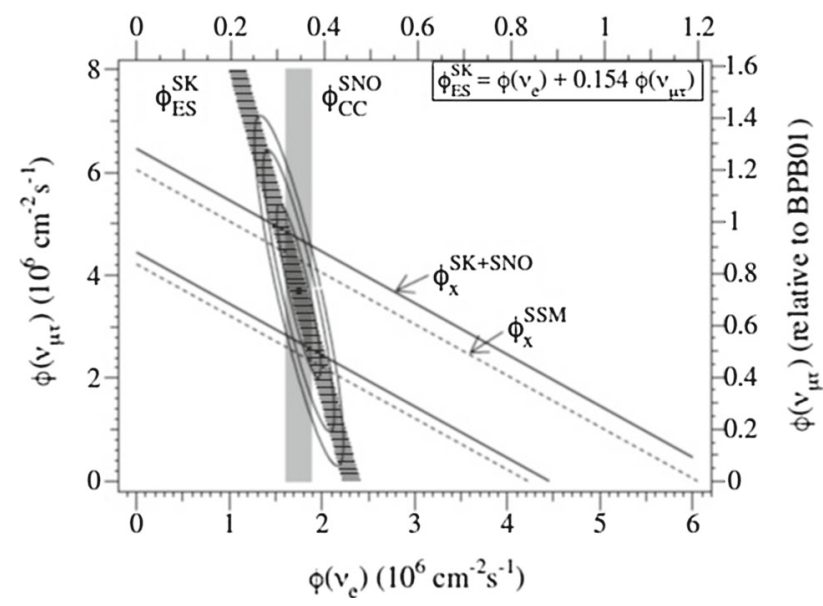

Fig. 11 The obtained $v_{e}$ and $v_{\mu . \tau}$ flux from the SNO charged current and Super-K $v+$ e scattering results (Fig. 3 from [60]). The apperance of non- $v_{e}$ component in the solar neutrino measured on the earth is demonstrated at $3.3 \sigma$

\section{Current situation of the solar neutrinos}

The solar neutrino data accumulated so far is 93,555 events from 5480 days of data taken between May 1996 and December 2017 (SK-I (1496 days), SK-II (791 days), SK-II (548 days), SK-IV(2860 days)). The directional distribution of those recoil electrons is shown in Fig. 12. It also demonstrates that the recently developed electron and gamma separation method, called multiple scattering goodness (MSG), works even in the low energy regions. MSG aims at identifying $\gamma$-ray background and eventually enhancing the electron sample and making it possible to extract solar neutrino events.

The energy threshold has improved as a function of time and has varied on the detector configuration and conditions. There is no doubt about the solar neutrino to oscillate, but precise data analysis with larger statistics with well controlled systematics would be needed to determine the oscillation parameter more precisely.

One noticeable observation so far is that the best obtained parameter value of the mass difference from the solar global analysis using all the solar neutrino experiments deviates
Fig. 12 Directional distribution of the solar neutrinos observed in Super-K shown below 7.5 $\mathrm{MeV}$ [64]. The event discrimination algorithm (MSG) works even in low energy regions down to $3.5 \mathrm{MeV}$ making recoil electron peaks prominent from backgrounds

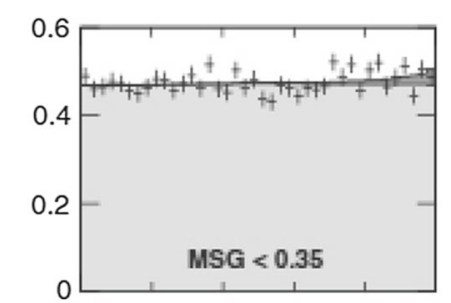

$3.49 \mathrm{MeV}<\mathrm{E}_{\mathrm{kin}}<3.99 \mathrm{MeV}$
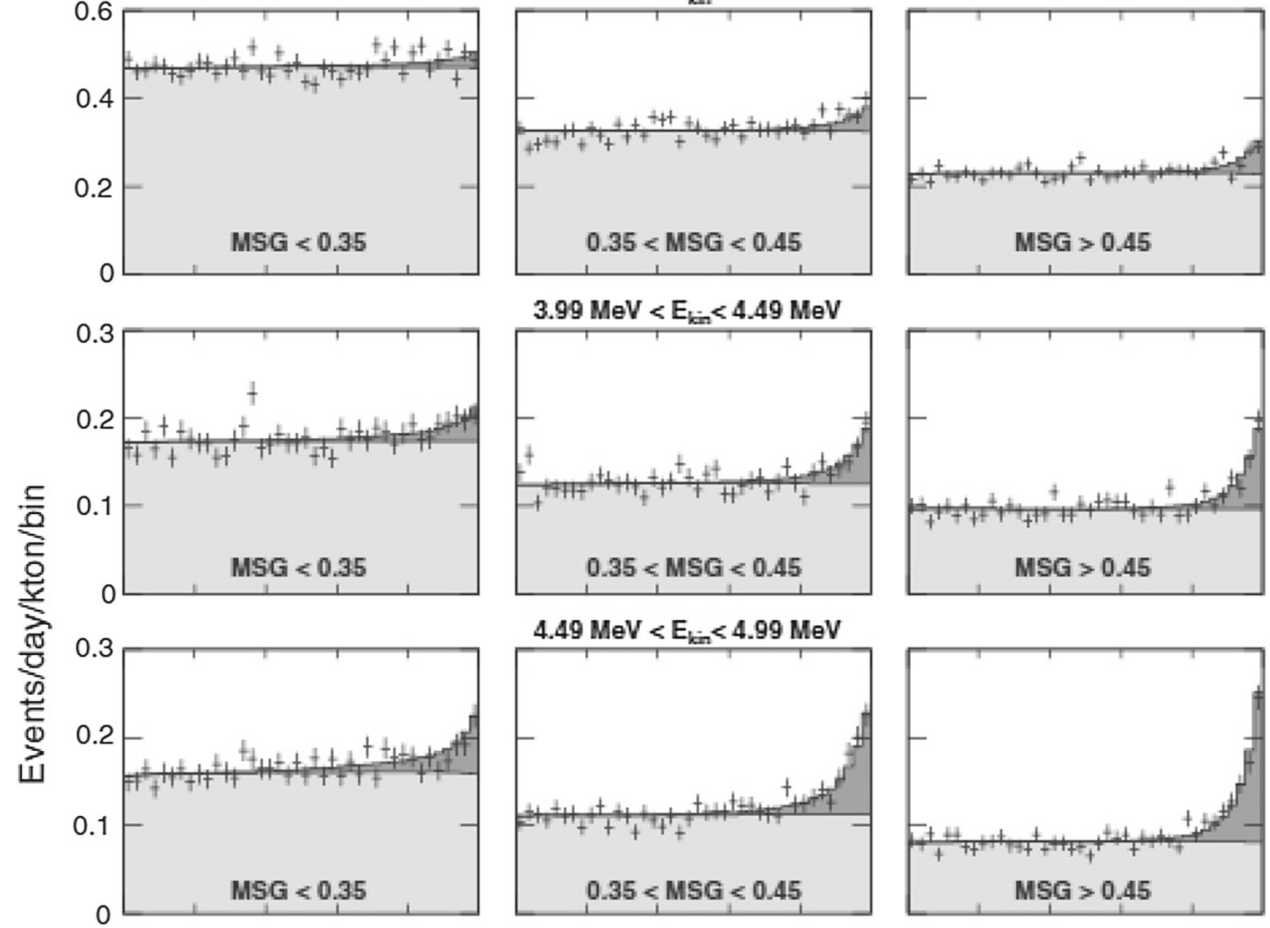

$3.99 \mathrm{MeV}<\mathrm{E}_{\mathrm{kin}}<4.49 \mathrm{MeV}$
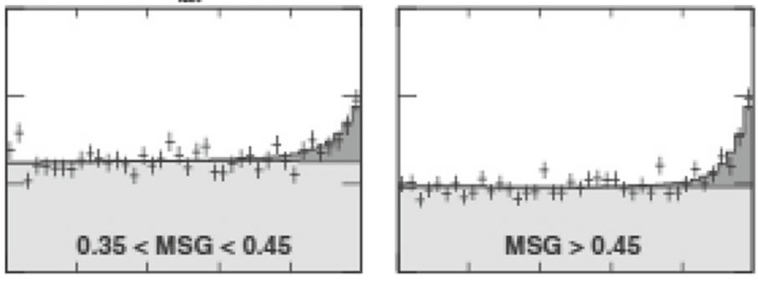

$4.49 \mathrm{MeV}<\mathrm{E}_{\mathrm{kin}}<4.99 \mathrm{MeV}$
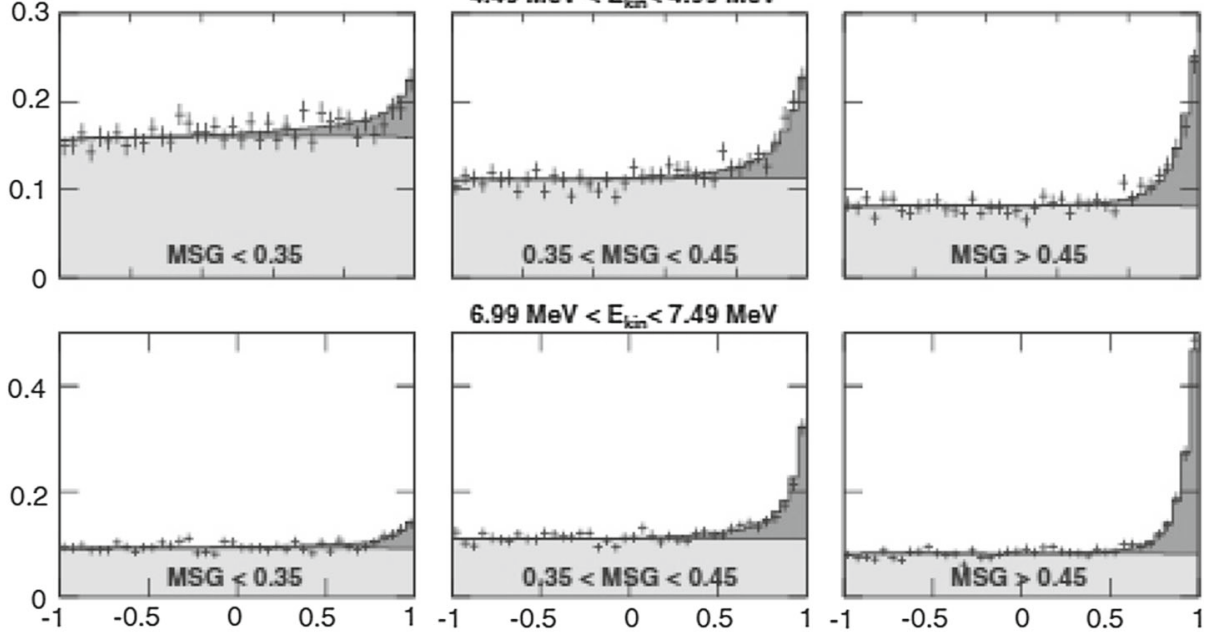


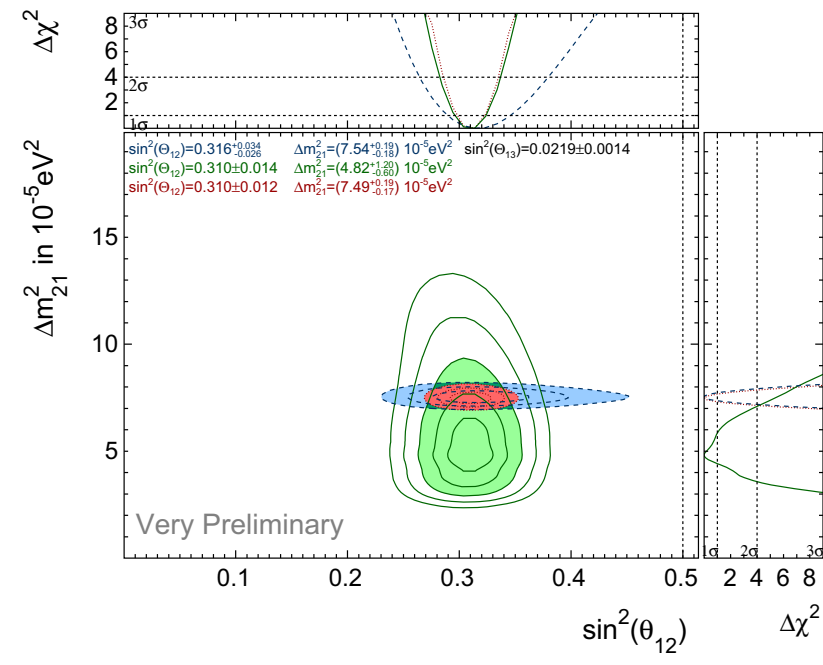

Fig. 13 Allowed parameter regions from the fits in the plane of $\sin ^{2} \theta_{12}$ and $\Delta m_{12}^{2}$. The green area shows the result from the solar global analysis and the blue one shows the KamLAND (long baseline reactor anti-neutrino oscillation experiment) results. The red colored region is obtained by combined all solar and KamLAND experiments. There is $\mathrm{a} \sim 2 \sigma$ difference between the two best fit values

from the value indicated from the KamLAND experiment - the long baseline reactor neutrino experiment - as seen in Fig. 13. It is important to settle this issue since the solar oscillation is in vacuum in the low energy region (pp- and ${ }^{7}$ Be-neutrinos), but affected by matter in the high energy region $\left({ }^{8} \mathrm{~B}\right.$-neutrinos). The mechanism of the oscillations is different from that of KamLAND that is basically vacuum oscillations of anti-neutrinos.

Those neutrinos produced at the center of the sun traverse the earth at the nighttime before reaching the terrestrial detector, but there are no obstacles between the sun and the detector at the daytime. The position of the sun, the hour, determines the zenith angle, the terrestrial matter density and length that the neutrinos pass through. Those neutrinos passing through the earth are influenced by earth's matter, and for most of the cases, regenerates $v_{e}^{\prime} s$ through the earth's matter. So the positive observation of the day night flux difference is direct evidence of the matter oscillation. The observed regeneration/survival probability gives another way to determine the oscillation parameters directly. We may also study some relevant effect in day/night flux difference like attenuation effect [61] which was recently pointed out.

The day/night asymmetry expected at the Super-K site is about $2 \sim 3 \%$. Precise measurements of the solar neutrino day-night effect were done using full SK-I, II and III data and 1664 days of data from SK-IV covering from May, 1996, till February, 2014 [62-64] as shown in Fig. 14. The average day/night rate ratios of difference and sum yielded

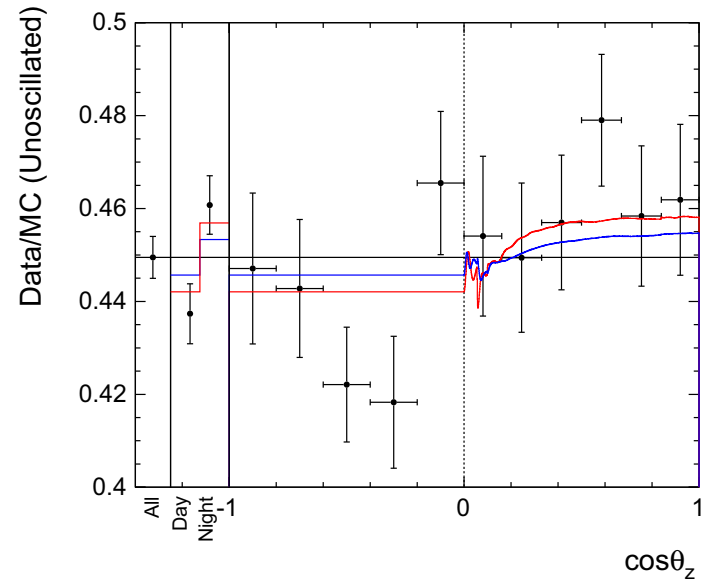

Fig. 14 The separated measurement of the solar day and night time flux in terms of the solar zenith angle distribution (only for SK-IV 1664 days of data) [64]. The red line and blue line are the expected flux for the best fit value of the solar global analysis and for that of the KamLAND analysis

$$
\begin{aligned}
A_{D N} & =\frac{2(\phi(\text { day })-\phi(\text { night }))}{(\phi(\text { day })+\phi(\text { night }))} \\
& =-(3.3 \pm 1.0(\text { stat }) \pm 0.5(\text { syst })) \%
\end{aligned}
$$

which is $2.9 \sigma$ evidence of the day/night flux difference. The uncertainty is still statistics dominated and we need further improvement. The systematic uncertainties of the flux were $3.2 \%, 2.1 \%$ and $1.7 \%$ for the SK-I, SK-III and SK-IV, respectively. Most of the systematic uncertainties cancelled by taking the flux ratios. The parameters determined solely from solar day/night effect are consistent with the solar neutrino global solution without the day/night effect. Therefore

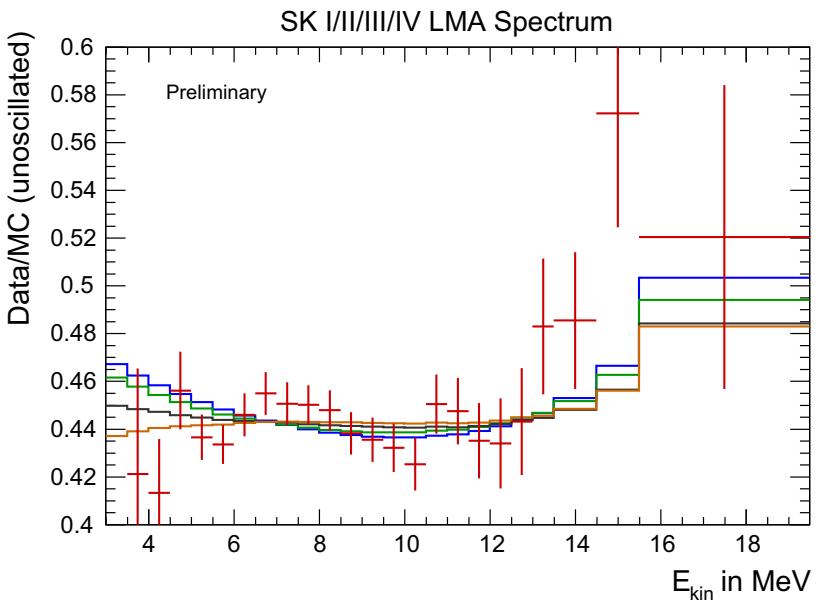

Fig. 15 The measured energy spectrum of all the Super-K phases combined. A clear upturn is not seen. The green line is expected from the solar global best and the blue line is that from KamLAND. The black and brown lines are the best fit lines for quadratic and exponential hypotheses 

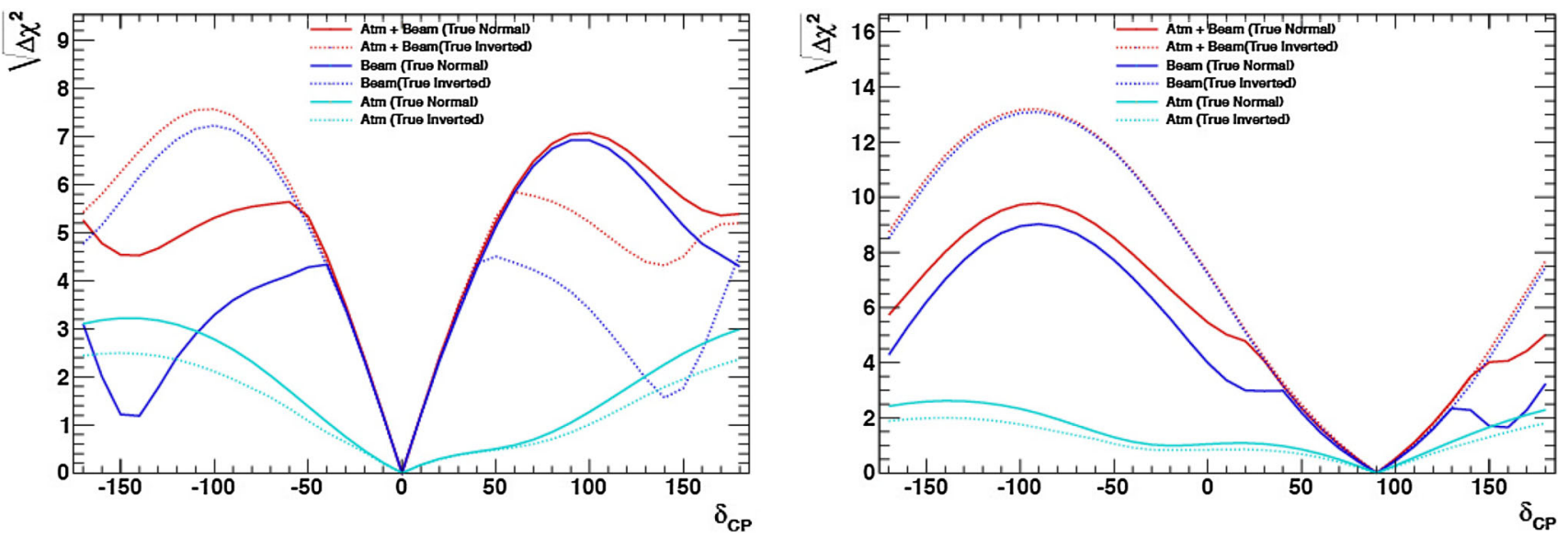

Fig. 16 The sensitivity for the CP phase assuming $0^{\circ}$ (left) and $90^{\circ}$ (right) [66]. The lines show the results from various combinations of the experiments. The atmospheric neutrino study alone in $\mathrm{HK}$ is not sufficient to conclude and combination with the beam neutrino study is neccesary

the further study of the day night flux difference is absolutely necessary.

For the large mixing angle solution, the low energy neutrinos, say below $1 \mathrm{MeV}$, undergo vacuum oscillation and the survival probability is about $\sim 70 \%$. In the high energy region, say above a few $\mathrm{MeV}$, undergo matter transition. There is no experiment covering the entire region of the transition between $1 \mathrm{MeV}$ and a few MeV. From the high energy side, we expect an upturn of the energy spectrum. However we have not observed yet [64] as shown in Fig. 15 including the last 2860 days of SK-IV data.

If we will not observe the upturn, then there may be new physics which is not explained by the standard model of elementary particle physics. It is then very important by continuously lowering the energy threshold to examine the effect, although Super-K is already running with the rather low energy threshold of $3.5 \mathrm{MeV}$ for the water Cherenkov detector.

\section{Summary and future}

The Super-Kamiokande experiment, the world largest low energy neutrino detector, started in 1996, with the aim of resolving neutrino problems, the solar neutrino puzzle and the atmospheric neutrino anomaly. Super-K also looked for proton decay, neutrino burst from supernovae. The discovery of neutrino oscillations was announced in 19982 years after the start of the operation and the evidence of the solar neutrino oscillation was shown by the data from SNO and Super-K in 2001. Detailed studies on the neutrino oscillations have been going on since the discoveries.

The remaining issues for atmospheric neutrinos are to determine the mass hierarchy, the octant of $\theta_{23}$ and the $\mathrm{CP}$ phase, if CP is violated. For the solar neutrino study the evi- dence of the matter effect, the day/night effect, need to be strengthened and the yet unresolved upturn issue needs to be settled. The upturn issue may be connected to new physics. During the last 23 years of operation, neither proton decay nor another neutrino burst from supernovae were not observed.

The continuous operation of Super-K may find some answers to the remaining problems, but the size of the SuperKamiokande detector is a limiting factor. For the CP study, for example, we are able to reach at most a $3 \sigma$ effect with the current configurations. In order to obtain a definitive answer for the CP problem, much larger detector is needed. For this reason the 256 kton water Cherenkov detector, HyperKamiokande $[65,66]$ with 8 times bigger fiducial mass than Super-K is planned in Japan.

Hyper-K with an accelerator beam data (T2HK) may provide $8 \sim 9 \sigma$ significance for the $\mathrm{CP}$ violation as shown in Fig. 16 in 10 years operation, if the $\mathrm{CP}$ phase is the current best fit value. The solar day/night effect is larger in the high energy region above $8 \sim 9 \mathrm{MeV}$. We may expect to measure with $5 \sim 6 \sigma$ sensitivity, although Hyper-K has a higher energy threshold than Super-K. We expect a proton decay sensitivity beyond $10^{35}$ years for $\mathrm{p} \rightarrow \mathrm{e}^{+} \pi^{0}$.

The Hyper-Kamiokande project was selected on the Roadmap2017 of the Japanese Ministry of Education, Culture, Sports, Technology and Science. We expect that the construction starts in April, 2020. It will take 8 years to construct and is expected to start in 2028. Hyper-K is an international collaboration consisting of 80 institutions from 17 countries. In conclusion, we are looking forward to a vigorous research program.

Data Availability Statement This manuscript has no associated data or the data will not be deposited. [Authors' comment: Data sharing not applicable to this article as no datasets were generated or analysed during the current study.] 
Open Access This article is distributed under the terms of the Creative Commons Attribution 4.0 International License (http://creativecomm ons.org/licenses/by/4.0/), which permits unrestricted use, distribution, and reproduction in any medium, provided you give appropriate credit to the original author(s) and the source, provide a link to the Creative Commons license, and indicate if changes were made.

Funded by SCOAP ${ }^{3}$.

\section{References}

1. Y. Watanabe et al., Proceedings of the Workshop on the Unified Theory and Baryon Number in the Universe, National Laboratory for High energy Physics, February, 1979. ed. O. Sawada, A. Sugamoto, KEK report, pp. 79-18 (1979)

2. Kamiokande proposal for the grant-in-aid for scientific research, September 1981 (in Japanese)

3. H. Georgi, S.L. Glashow, Phys. Rev. Lett. 32, 438 (1974)

4. H. Georgi, H.R. Quinn, S. Weinberg, Phys. Rev. Lett. 33, 451 (1974)

5. F. Reines, M.F. Crouch, Phys. Rev. Lett. 32, 493 (1974)

6. M. Koshiba, Kamioka Nucleon Decay Experiment. In Proceedings of ICOBAN84, p. 69 (1984)

7. Kamiokande Collaboration (M. Koshiba for the collaboration), 22-kton Water Cherenkov Detector (JACK). In Proceedings of ICOBAN84, p. 230 (1984)

8. K. Hirata et al., Phys. Rev. Lett. 58, 1490 (1987)

9. K.S. Hirata et al., Phys. Rev. Lett. 63, 16 (1989)

10. E. Eguchi et al., Phys. Rev. Lett. 90, 021802 (2003)

11. S. Fukuda et al., Nucl. Instrum. Methods A 501, 418 (2003)

12. Z. Maki, M. Nakagawa, S. Sakata, Prog. Theor. Phys. 28, 870 (1962)

13. B. Pontecorvo, Z. Eksp, Teor. Fiz 53, 1717 (1967)

14. L.-L. Chau, W.-Y. Keung, Phys. Rev. Lett. 53, 1802 (1984)

15. C. Patrignanj et al., Particle Data Group. Chin. Phys. C 40, 100001 (2016)

16. D. Maurin et al., Astron. Astrophys. A 32, 569 (2014)

17. K. Abe et al., Phys. Rev. Lett. 108, 051102 (2012)

18. Y.S. Yoon et al., Astrophys. J. 728, 122 (2011)

19. O. Adriani et al., Astrophys. J. 765, 91 (2013)

20. M. Aguilar et al., Phys. Rev. Lett. 114, 171103 (2015)

21. Y. Futagami et al., Phys. Rev. Lett. 82, 5194 (1999)

22. E. Richard et al., Phys. Rev. D 94, 052001 (2016)

23. S. Fukuda et al., Nucl. Instrum. Methods A 501, 418 (2003). and references therein
24. K. Abe et al., Phys. Rev. D 92, 112003 (2015)

25. S. Kasuga et al., Phys. Lett. B 374, 238 (1996)

26. K.S. Hirata et al., Phys. Lett. 205, 416 (1988)

27. S.M. Bilenky, B. Pontecorvo, Phys. Rep. 41, 225 (1978)

28. M. Aglietta et al., Europhys. Lett. 8, 611 (1989)

29. K. Daum et al., Z. Phys. C 66, 417 (1995)

30. W.W.M. Allison et al., Phys. Lett. B 391, 491 (1997)

31. W.W.M. Allison et al., Phys. Lett. B 449, 137 (1999)

32. R. Becker-Szendy et al., Phys. Rev. D 46, 3720 (1992)

33. Y. Fukuda et al., Phys. Lett. B 335, 237 (1994)

34. Y. Fukuda et al., Phys. Rev. Lett. 81, 1562 (1998)

35. Y. Fukuda et al., Phys. Lett. B 433, 9 (1998)

36. Y. Fukuda et al., Phys. Lett. B 436, 33 (1998)

37. Y. Fukuda et al., Phys. Rev. Lett. 82, 2644 (1999)

38. Y. Ashie et al., Phys. Rev. D 71, 112005 (2005)

39. J. Hosaka et al., Phys. Rev. D 74, 032002 (2006)

40. R. Wendell et al., Phys. Rev. D 81, 092004 (2010)

41. K. Abe et al., Phys. Rev. D 97, 072001 (2018)

42. O.L.G. Peres, A.Yu. Smirnov, Phys Lett. B 456, 204 (1999), and references therein

43. W.C. Haxton, A.M. Serenelli, arXiv:0805.2013v1

44. M. Asplund et al., Ann. Rev. Astron. Astrophys. 47, 481 (2009)

45. N. Grevesse, A.J. Sauval, Spc. Sci. Rev. 85, 161 (1998)

46. J.N. Bahcall, S. Basu, M. Pinsonneault, Phys. Lett. B 433, 1 (1998)

47. A.M. Serenelli et al., Astron. Phys. J. 743, 24 (2011)

48. R. Davis et al., Phys. Rev. Lett. 20, 1205 (1968)

49. B.T. Cleveland et al., ApJ. 496, 505 (1998)

50. L. Wolfenstein, Phys. Rev. D 17, 2369 (1978)

51. S.P. Mikheev, A.Y. Smirnov, Nuovo Cim. C 9, 17 (1986)

52. J.N. Abdurashitov et al., Phys. Lett. B 328, 234 (1994)

53. P. Anselmann et al., Phys. Lett. B 285, 376 (1992)

54. W. Hampel et al., Phys. Lett. B 388, 364 (1996)

55. Y. Fukuda et al., Phys. Rev. Lett. 81, 1158 (1998); Erratum-ibid. 81, 4279 (1998)

56. Y. Fukuda et al., Phys. Rev. Lett. 82, 1810 (1999)

57. Y. Fukuda et al., Phys. Rev. Lett. 82, 2430 (1999)

58. S. Fukuda et al., Phys. Rev. Lett. 86, 5651 (2001)

59. S. Fukuda et al., Phys. Rev. Lett. 86, 5656 (2001)

60. Q.R. Ahmad et al., Phys. Rev. Lett. 87, 071301 (2001)

61. A.N. Ioannisian, A.Y. Smirnov, Phys. Rev. D 96, 083009 (2017)

62. M.B. Smy et al., Phys. Rev. D 69, 011104 (2004)

63. A. Renshaw et al., Phys. Rev. Lett. 112, 091805 (2014)

64. K. Abe et al., Phys. Rev. D 94, 052010 (2016)

65. K. Ape et al., Prog. Theor. Exp. Phys. 2015, 053 C02 (2015)

66. K. Abe et al., Prog. Theor. Exp. Phys. 2018, 063 C01 (2018) 